\title{
Distribution of Serotonin-Immunoreactive Neurons in the Brain and Gnathal Ganglion of Caterpillar Helicoverpa armigera
}

\author{
Qing-Bo Tang, Wei-Wei Song, Ya-Jun Chang, Gui-Ying Xie, Wen-Bo Chen* \\ and Xin-Cheng Zhao*
}

Department of Entomology, College of Plant Protection, Henan Agricultural University, Zhengzhou, China

Serotonin (5-hydroxytryptamine, 5-HT) is an important biogenic amine that acts as a neural circuit modulator. It is widespread in the central nervous system of insects. However, little is known about the distribution of serotonin in the nervous system of the cotton bollworm Helicoverpa armigera. In the present study, we performed immunohistochemical experiments with anti-serotonin serum to examine the distribution of serotonin in the central nervous system of $\mathrm{H}$. armigera larvae. We found about 40 serotonin-immunoreactive neurons in the brain and about 20 in the gnathal ganglion. Most of these neurons are wide-field neurons giving rise to processes throughout the neuropils of the brain and the gnathal ganglion. In the central brain, serotonin-immunoreactive processes are present bilaterally in the tritocerebrum, the

OPEN ACCESS

Edited by:

Francesco Fornai, University of Pisa, Italy

Reviewed by: Adhil Bhagwandin, University of Cape Town, South Africa

Wei Xu,

Murdoch University, Australia

${ }^{*}$ Correspondence: Xin-Cheng Zhao xincheng@henau.edu.cn Wen-Bo Chen wenbochen@henau.edu.cn

Received: 13 February 2019 Accepted: 14 May 2019

Published: 28 May 2019

Citation: Tang $Q-B$, Song $W-W$, Chang $Y-J$, Xie G-Y, Chen W-B and Zhao X-C (2019) Distribution of Serotonin-Immunoreactive Neurons in the Brain and Gnathal Ganglion of

Caterpillar Helicoverpa armigera. Front. Neuroanat. 13:56. doi: 10.3389/fnana.2019.00056 deutocerebrum, and major regions of the protocerebrum, including the central body (CB), lateral accessory lobes (LALS), clamps, crepine, superior protocerebrum, and lateral protocerebrum. The CB, anterior ventrolateral protocerebrum (AVLP), and posterior optic tubercle (POTU) contain extensive serotonin-immunoreactive process terminals. However, the regions of mushroom bodies, the lateral horn, and protocerebral bridges (PBs) are devoid of serotonin-immunoreactivity. In the gnathal ganglion, the serotonin-immunoreactive processes are also widespread throughout the neuropil, and some process projections extend to the tritocerebrum. Our results provide the first comprehensive description of the serotonergic neuronal network in $\mathrm{H}$. armigera larvae, and they reveal the neural architecture and the distribution of neural substances, allowing us to explore the neural mechanisms of behaviors by using electrophysiological and pharmacological approaches on the target regions.

Keywords: Helicoverpa armigera, serotonin, immunoreactivity, wide-field neurons, commissure, neuropils, brain, gnathal ganglion

\section{INTRODUCTION}

Serotonin (5-hydroxytryptamine, 5-HT) is a biogenic amine that is widely present in both invertebrate and vertebrate animal species (Vleugels et al., 2015). In insects, as in vertebrates and other invertebrates, serotonin is an important monoamine neurotransmitter that functions as a neural circuit modulator (Nässel, 1988; Qi et al., 2014; Vleugels et al., 2015). Antiserum against serotonin has been available for three decades, and the immunohistochemical experiments to 
investigate the distribution of serotonin in the nervous system have been performed in a large number of insect species, including locusts, mantes, cockroaches, bugs, beetles, ants, wasps, bees, flies, mosquitoes, and moths (Bishop and O'Shea, 1983; Tyrer et al., 1984; Lange et al., 1988; Nässel, 1988; Homberg and Hildebrand, 1989a,b; Breidbach, 1990; Boleli and PaulinoSimões, 1999; Leitinger et al., 1999; Settembrini and Villar, 2004; Dacks et al., 2006; Liu et al., 2011; Huser et al., 2012; van der Woude and Smid, 2017). Serotonin is distributed throughout the nervous system, including both peripheral and central regions. Physiological and behavioral experiments have demonstrated that serotonin is involved in vision (Paulk et al., 2009), olfaction (Linn and Roelofs, 1986; Gatellier et al., 2004; Dacks et al., 2008; Kloppenburg and Mercer, 2008; Ellen and Mercer, 2012; Muscedere et al., 2012), hearing (Andrés et al., 2016), feeding (Ali, 1997; Kaufmann et al., 2004; Orchard, 2006; Haselton et al., 2009; French et al., 2014; Schoofs et al., 2018), circadian behavior (Hinks, 1967; Page, 1987; Cymborowski, 1998; Chen et al., 1999; Tomioka, 1999; Saifullah and Tomioka, 2002; Yuan et al., 2005; Giese et al., 2018), aggregation (Anstey et al., 2009; Rogers and Ott, 2015), and learning and memory (Sitaraman et al., 2008, 2012; Fernández et al., 2012).

The cotton bollworm Helicoverpa armigera (Hübner; Lepidoptera: Noctuidae), is an important agricultural pest in the world, feeding on more than 160 plant species (Fitt, 1989; $\mathrm{Ma}$ et al., 2016). The damage of $H$. armigera to plants occurs during its larval stage. Feeding behavior of $H$. armigera larvae is mediated to a large extent by the gustatory sensillum, which detects palatable and unpalatable compounds in plants to acquire nutrients and avoid the toxins (Zhou et al., 2010). Behavioral and gustatory electrophysiological studies revealed that feeding experiences could induce changes in feeding preference of H. armigera for host plants (Zhou et al., 2010; Ma et al., 2016; Wang et al., 2017). Such feeding preference and plasticity is mediated by the signal transduction of the gustatory pathway and the modulation of the neural substance in the central nervous system (Glendinning et al., 1999, 2001). However, to date little is known about the neural substances in the nervous system of $H$. armigera.

In the present study, we performed immunohistochemistry with anti-serotonin serum to examine the distribution of serotonin in the central nervous system of $H$. armigera larvae. We provide the first comprehensive description of the serotonergic neuronal network in $H$. armigera larvae, which is the basic knowledge about the neural architecture and the distribution of neural substance and improves our understanding of the neural mechanism of behaviors, such as, host selection, navigation, and feeding preference and plasticity. In addition, the findings are essential to develop novel methods to control the pest by modulating the insect behaviors with the neural substance of serotonin.

\section{MATERIALS AND METHODS}

\section{Insects}

Larvae of $H$. armigera were reared on an artificial diet in the laboratory under $16 / 8 \mathrm{~h}$ light/dark, at $27^{\circ} \mathrm{C}$ and $70 \%$ relative humidity. Two-day-old 5th instar larvae were used for the experiments. No permission from the ethics committee is required for experiments on $H$. armigera according to the laws on animal welfare in China.

\section{Immunocytochemistry for Serotonin and Synapsin}

Brains and gnathal ganglia of $H$. armigera larvae were dissected out in fresh Ringer's solution [150 mM NaCl, $3 \mathrm{mM}$ $\mathrm{CaCl}_{2}, 3 \mathrm{mM} \mathrm{KCl}, 25 \mathrm{mM}$ Sucrose, and $10 \mathrm{mM} \mathrm{N}$-tris (hydroxymethyl)-methyl-2-amino-ethanesulfonic acid, $\mathrm{pH}$ 6.9] on ice. Immunostaining with anti-serotonin was performed to examine the distribution of serotonin-immunoreactive neurons in the brain and the gnathal ganglion. To visualize the outline of the neuropil structure, immunostaining with anti-synapsin was also performed. The immunostaining of serotonin and synapsin was conducted following previously described procedures (Zhao et al., 2016). The dissected brains and gnathal ganglia were fixed in $4 \%$ paraformaldehyde for $1-2 \mathrm{~h}$ at room temperature, and they were rinsed four times with phosphate-buffered saline (PBS; $684 \mathrm{mM} \mathrm{NaCl}, 13 \mathrm{mM} \mathrm{KCl}, 50.7 \mathrm{mM} \mathrm{Na}_{2} \mathrm{HPO}_{4}$, $5 \mathrm{mM} \mathrm{KH} \mathrm{PO}_{4}, \mathrm{pH}$ 7.4) for $15 \mathrm{~min}$. To minimize the non-specific staining, the rinsed brains and gnathal ganglia were pre-incubated in 5\% normal goat serum (NGS, Sigma, St. Louis, MO, USA) in PBS (0.1 M, pH 7.4) containing $0.5 \%$ Triton X-100 (PBSX) for $3 \mathrm{~h}$ at room temperature. Next, the samples were incubated with anti-serotonin serum (1:5,000; Immunostar Inc., Hudson, WI, USA) and anti-SYNORF1 serum (1:200; Developmental Studies Hybridoma Bank, University of Iowa, Iowa City, IA, USA) in PBSX containing 5\% NGS for 5 days at $4^{\circ} \mathrm{C}$. The specificity of anti-SYNORF1 had been confirmed by both western blot and immunohistochemistry (Godenschwege et al., 2004). The labeling of the synaptic neuropil with this antibody had been reported previously in a large number of insect species, including heliothine moths (Zhao et al., 2016). The specificity of the anti-serotonin had been tested in heliothine moth by preadsorption of lyophilized serotonin creatine sulfate coupled to bovine serum albumin (Immunostar) at a concentration of $20 \mu \mathrm{g} / \mathrm{ml}$ (Zhao and Berg, 2009). The preadsorption abolished all immunostaining with anti-serotonin serum in the brain of heliothine moths. Following six rinse in PBS for $20 \mathrm{~min}$, the brains and gnathal ganglia were incubated in secondary Cy2-conjugated goat anti-mouse antibodies (1:500; Invitrogen, Eugene, OR, USA) and Cy5-conjugated goat anti-rabbit antibodies (1:500; Invitrogen) in PBSX for 3 days at $4^{\circ} \mathrm{C}$ in the dark. After being rinsed in PBS $6 \times 20 \mathrm{~min}$, the brains and gnathal ganglia were dehydrated in an ethanol series (50\%, 70\%, 90\%, 96\%, and $2 \times 100 \%$, each $10 \mathrm{~min}$ ), cleared in methyl salicylate, and mounted in Permount.

\section{Image Acquisition, Three-Dimensional Reconstruction, and Image Processing}

The images of serial confocal stacks were acquired by using a confocal laser scanning microscope (LSM710, META Zeiss, Jena, Germany) with an objective of $10 \times$ (PlanNeofluar $10 \times / 0.3)$ and $20 \times$ (Plan-Neofluar $20 \times / 0.51$ ). 

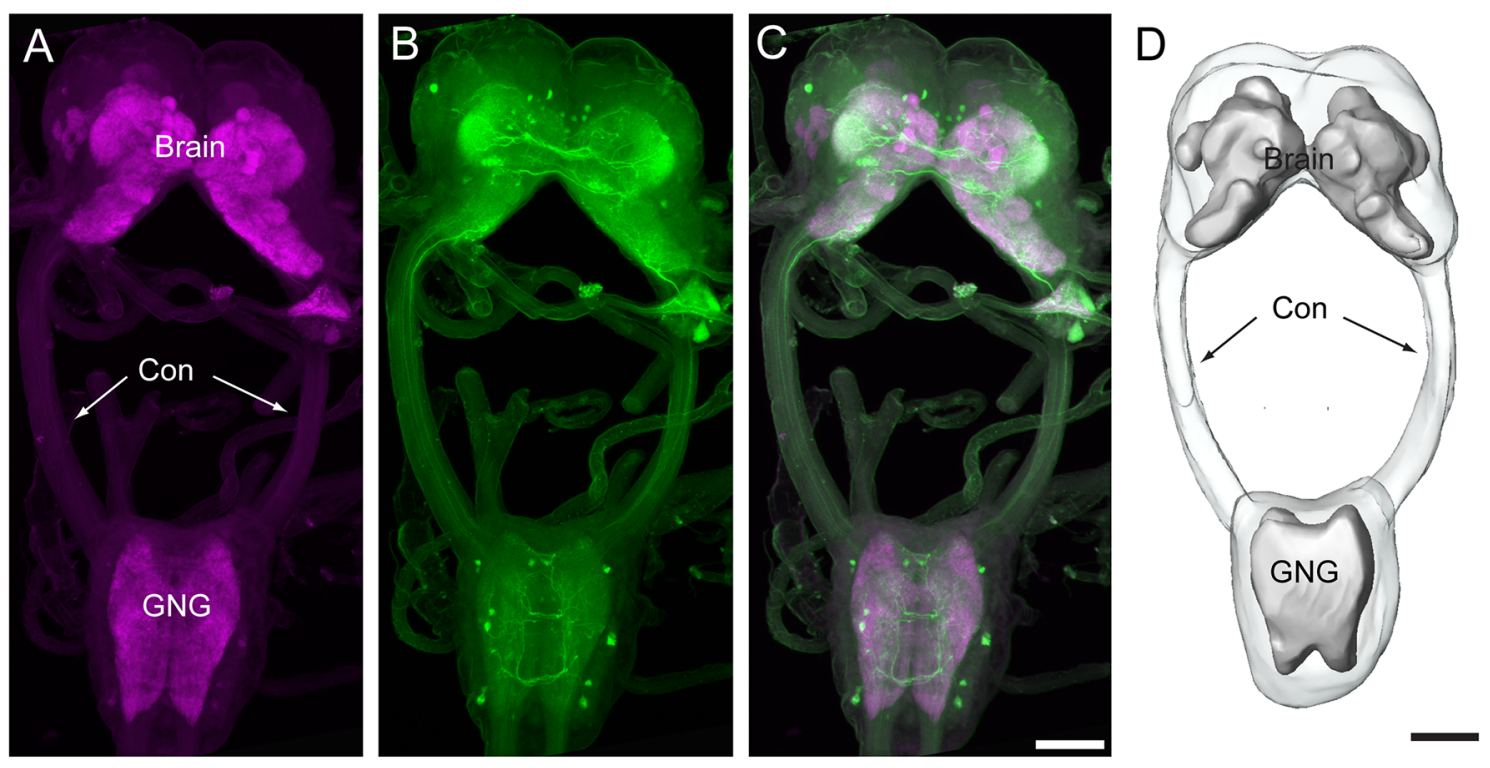

FIGURE 1 | Confocal images and three-dimensional reconstruction of the brain and the gnathal ganglion of Helicoverpa armigera larvae. (A) Confocal image showing the neuropils of the brain and the gnathal ganglion. (B) Confocal image showing the serotonin-immunoreactive neurons in the brain and the gnathal ganglion. (C) Merged confocal image showing the neuropil (magenta) and the serotonin-immunoreactive neurons (green). (D) Three-dimensional reconstruction of the brain and the gnathal ganglion. Con, connective; GNG, gnathal ganglion. Scale bars, $100 \mu \mathrm{m}$.

To excite Cy2 and Cy5, a 488-nm 1 Argon laser and a $633 \mathrm{~nm} \mathrm{HeNe}$ laser were used, respectively. The resolutions of images are $1,024 \times 1,024$ pixels and the intervals are $2-3 \mu \mathrm{m}$.

To create the three-dimensional models of brains, gnathal ganglia, cell bodies, and thick fiber of serotonin-immunoreactive neurons, the confocal stacks were imported into the visualization software AMIRA (AMIRA 5.3, Visage Imaging, Fürth, Germany). The Segmentation editor tool was used to label the neuropils and the Skeleton tree was used to trace the thick neuron fibers (Zhao et al., 2017).

Necessary adjustments for brightness and contrast of the images were made in Adobe Photoshop, and the final figure panels were edited in Adobe Illustrator CS2 (Adobe System, San Jose, CA, USA). Terminology and abbreviations for neuropil structures suggested by Ito et al. (2014) were used for the brain and the gnathal ganglion of $H$. armigera larvae. The axis of neuropil is taken as the axis of the insect body. The orientation of the larval brain is about $90^{\circ}$ different from that of adult insects, however, the neuropil structures in the larval brain are given the same names as the corresponding structures in adult insects, regardless of the orientation.

\section{RESULTS}

In total, immunocytochemical experiments for synapsin and serotonin were performed on 24 preparations. Of these, 16 were successfully stained, and seven were used to obtain the confocal images and to count the number of serotonin-immunoreactive neurons in the brain and the gnathal ganglion. The brains and gnathal ganglia of $H$. armigera larvae were connected by a pair of circumesophageal connectives (Figure 1). The serotoninimmunoreactive neurons were reliably distinguished by their cell body positions in the cell body rind of the brain and the gnathal ganglion. The nomenclature of neurons used in this report is based on the cell body position.

\section{General Anatomy of the $\boldsymbol{H}$. armigera Larval Brain}

The brain of $H$. armigera larvae contains three distinct neuromeres, i.e., tritocerebrum (TR), deutocerebrum (DE), and protocerebrum (PR; Tang et al., 2014). The tritocerebrum is located most ventrally in the brain, and the deutocerebrum is located above the tritocerebrum (Figures 2, 3). The deutocerebrum consists of the antennal lobe (AL) and the antennal mechanosensory and motor center (AMMC; Figures 2, 3). The protocerebrum is the largest part of the brain and is located dorsally. Within the protocerebrum, the neuropils of the optic lobe (OL), mushroom body (MB), central body $(\mathrm{CB})$, protocerebral bridge $(\mathrm{PB})$, and lateral accessory lobe (LAL) were most prominent and easiest to identify (Figures 2, 3). In larvae the OL is located most lateral of the protocerebrum, the $\mathrm{CB}$ is in the center, and the calyx (CA) of $\mathrm{MB}$ is located most dorsally (Figures $\mathbf{2 A - C}$ ). By referring to the detailed three-dimensional reconstructed brain maps of the fruit fly Drosophila melanogaster (Ito et al., 2014), a large number of homologous neuropils in the remaining part of the central brain were also identified. The superior neuropils contained the superior medial protocerebrum (SMP), the superior intermediate protocerebrum (SIP), the superior lateral protocerebrum (SLP), and the LH (Figures 2, 3B,C). The lateral neuropils comprise the inferior lateral protocerebrum 


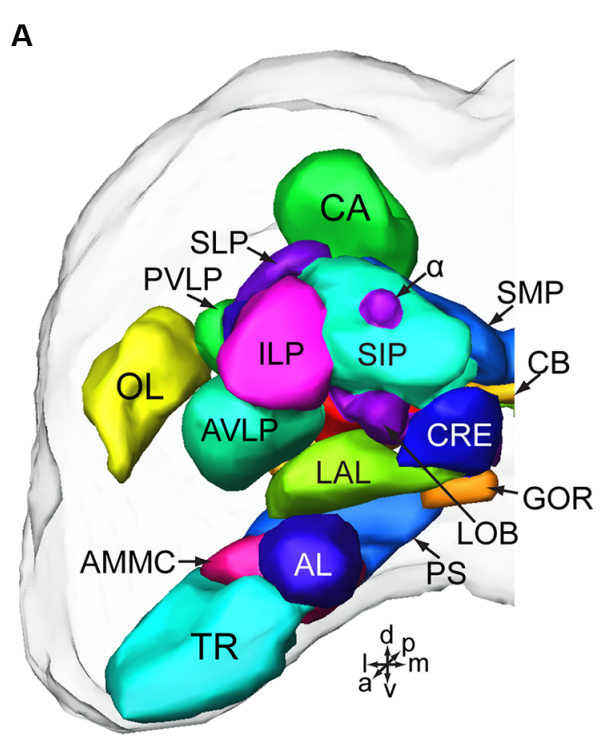

C

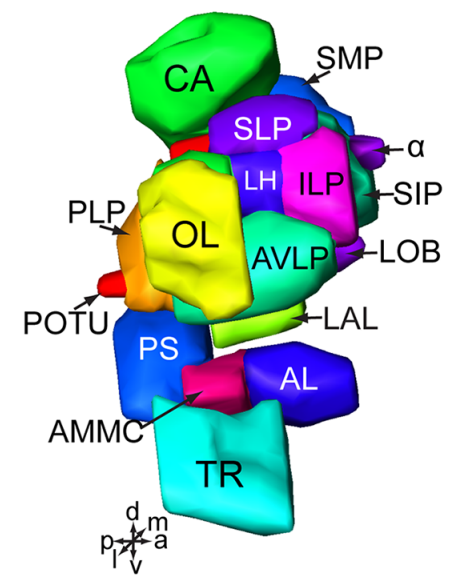

B

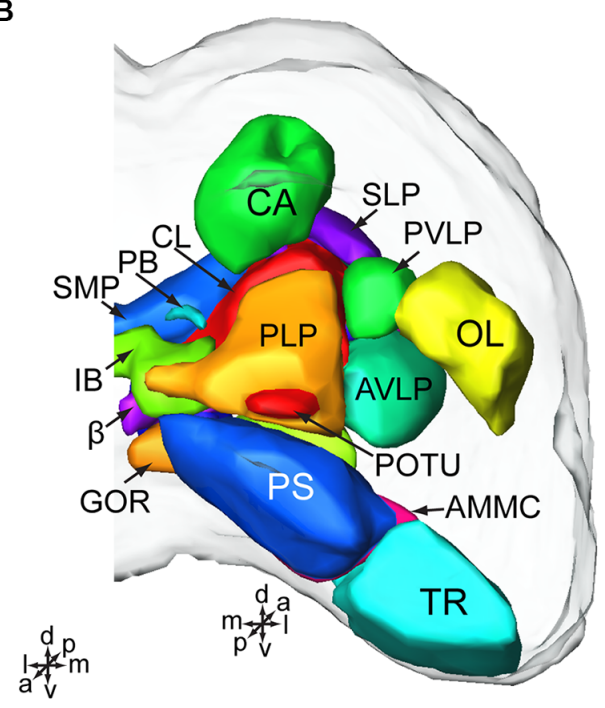

D

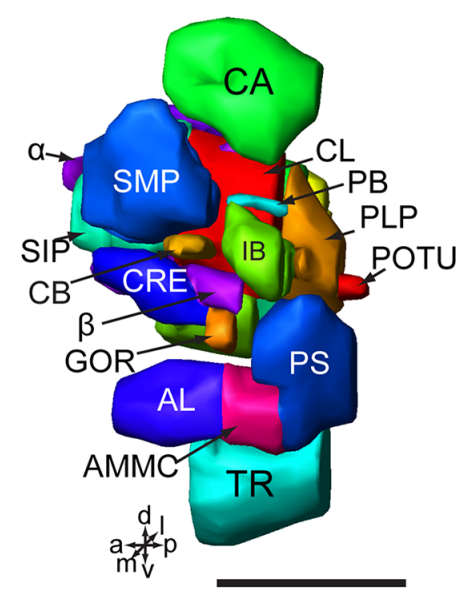

FIGURE 2 | Brain composition of H. armigera larvae. (A) Three-dimensional reconstuctions of one brain hemisphere in frontal view; (B) in posterior view; (C) in lateral view; (D) in sagittal view. $\alpha$, alpha lobe; AL, antennal lobe; AMMC, antennal mechanosensory and motor center; AVLP, anterior ventrolateral protocerebrum; $\beta$, belta lobe; CA, calyx; CB, central body CL, clamp; CRE, crepine; GOR, gorget; IB, inferior bridge; ILP, inferior lateral protocerebrum; LAL, lateral accessory lobe; LH, lateral horn; LOB, mushroom body lobes; OL, optic lobe; PB, protocerebral bridge; PLP, posterior lateral protocerebrum; POTU, posterior optic tubercle; PS, posterior slope; PVLP, posterior ventrolateral protocereburm; SIP, superior intermediate protocerebrum; SLP, superior lateral protocerebrum; SMP, superior medial protocerebrum; TR, tritocerebrum. Directions: a, anterior; d, dorsal; I, lateral; m, medial; p, posterior, v, ventral. Scale bar, 100 m.

(ILP), the anterior ventrolateral protocerebrum (AVLP), the posterior ventrolateral protocerebrum (PVLP), the posterolateral protocerebrum (PLP), and the posterior optic tubercle (POTU; Figures 2, 3C,E). The inferior neuropils include the crepine (CRE), clamp (CL), and inferior bridge (IB; $\mathrm{x})$. The ventromedial neuropils include the posterior slope (PS) and the gorget (GOR; Figures 2, 3F,H).

\section{Number of Serotonin-Immunoreactive Cell Bodies in the $\boldsymbol{H}$. armigera Larval Brain}

Serotonin-immunoreactivity was detected in a large area of the brain in $H$. armigera larvae (Figures 3, 4). All detected cell bodies were labeled and counted, and thick fibers were traced (Figure 4;
Table 1). There were about 30-38 serotonin-immunoreactive cell bodies distributed singly or in clusters in both hemispheres of the brain (Figures 4A-C; Table 1). The cluster of PR-M contained the largest number of serotonin-immunoreactive cell bodies, about 10 in each hemisphere, distributed in the medial region of the posterior protocerebrum, ventrolateral to the calyx (Figures 4B,C; Table 1). The cluster of PR-LD, contained about three cell bodies in each hemisphere, located dorsally to the lateral protocerebral neuropil (Figures 4B,C; Table 1). The serotonin staining in these cell bodies was weak. The cluster of PR-L contained one cell body in each hemisphere, located laterally to the lateral protocerebral neuropil (Figure 4B; Table 1). Among seven preparations counted, 

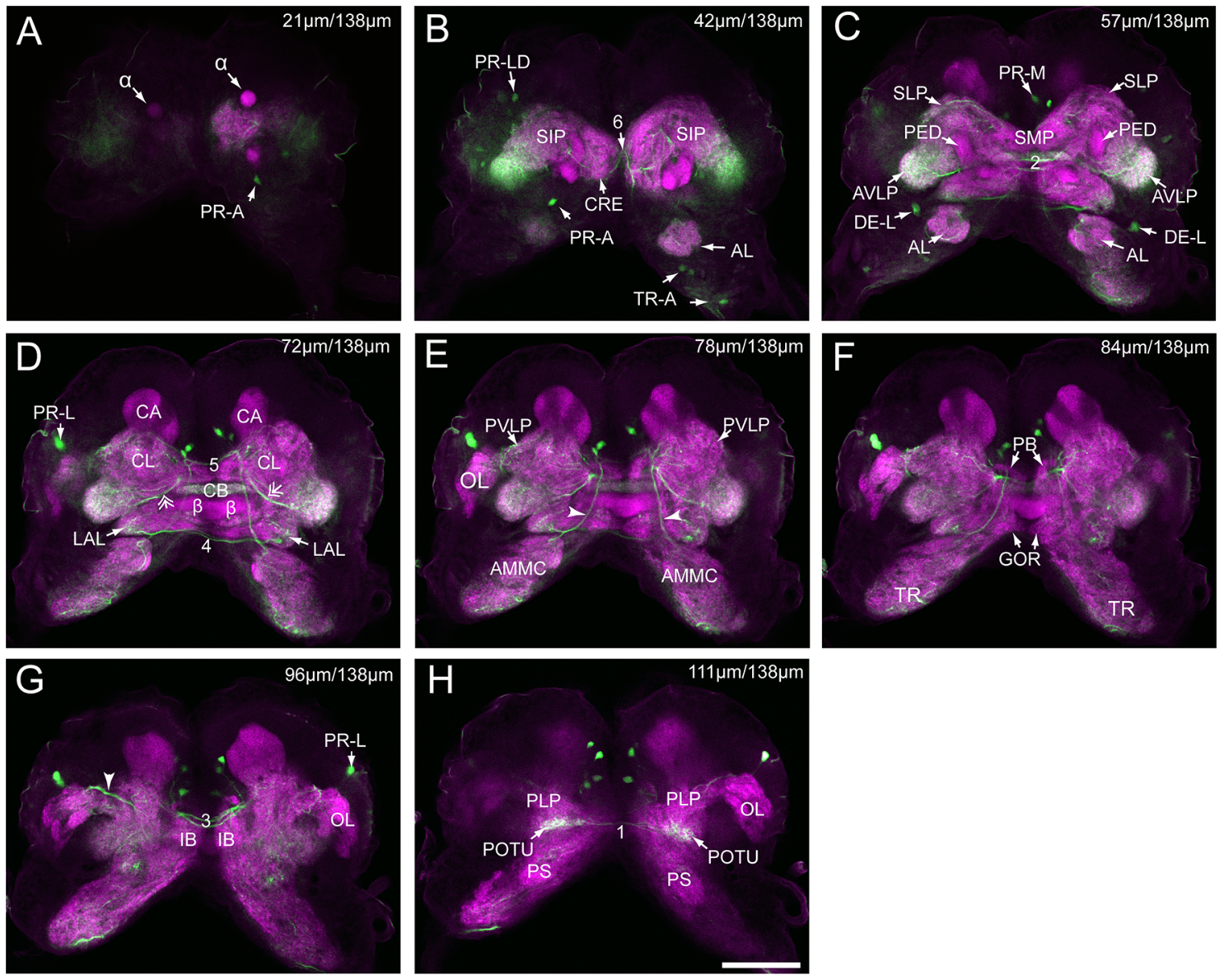

FIGURE 3 | Serial confocal images from anterior to posterior showing the serotonin- immunoreactive neurons (green) and the brain neuropils (magenta) of Helicoverpa armigera larvae. (A) Confocal image of the brain section at a depth of $21 \mu \mathrm{m}$ of total $138 \mu \mathrm{m}$; (B) at $42 \mu \mathrm{m}$; (C) at $57 \mu \mathrm{m}$; (D) at $72 \mu \mathrm{m}$. Double arrows indicate the processes from the cells in the cluster of PR-M extending to the AVLP; (E) at $78 \mu \mathrm{m}$. Arrowheads indicate the processes of the cell in the cluster of DE-L; (F) at $84 \mu \mathrm{m}$; (G) at $96 \mu \mathrm{m}$. Arrowhead indicates the processes of cells in the cluster of PR-L; (H) at 111 $\mu \mathrm{m}$. $\alpha$, alpha lobe; AL, antennal lobe; AMMC, antennal mechanosensory and motor center; AVLP, anterior ventrolateral protocerebrum; $\beta$, belta lobe; CA, calyx; CB, central body; CL, clamp; CRE, crepine; GOR, gorget; IB, inferior bridge; ILP, inferior lateral protocerebrum; LAL, lateral accessory lobe; LOB, mushroom body lobes; OL, optic lobe; PB, protocerebral bridge; PLP, posterior lateral protocerebrum; POTU, posterior optic tubercle; PS, posterior slope; PVLP, posterior ventrolateral protocereburm; SIP, superior intermediate protocerebrum; SLP, superior lateral protocerebrum; SMP, superior medial protocerebrum; TR, tritocerebrum. DE-L, PR-A, PR-L, PR-LD, PR-M, and TR-A are cell clusters. 1, the posterior protocerebral commissure linking the POTU; 2, the median protocerebral commissure linking the CB and bilateral SIP; 3 , the posterior great commissure linking the bilateral AVLP and the CL; 4, the LAL commissure; 5 , the dorsal protocerebral commissure linking the bilateral AL and the SLP; 6 , the anterior protocerebral commissure linking the bilateral tritocerebrum. Scale bar, $100 \mu \mathrm{m}$.

however, there was one individual preparation containing two cell bodies in one cluster of PR-L. The cluster of PR-A is located anteriorly to the protocerebrum and dorsally to the AL (Figure 4B). We observed a single brightly stained serotoninimmunoreactive cell body in each hemisphere in the cluster of PR-A (Figures 4B,C; Table 1). In some preparations, however, we observed an additional weakly stained cell in the cluster of PR-A (Table 1).

The cluster of DE-L, located laterally to the AL, contained a single serotonin-immunoreactive cell body in each hemisphere (Figure 4B; Table 1). In the tritocerebrum, we observed three weakly stained serotonin-immunoreactive cell bodies in each hemisphere in the cluster of TR-A, located anteriorly to the tritocerebrum (Figure 4B; Table 1). In some preparations, we did not detect serotonin-immunoreactive the cells in the cluster of TR-A.

\section{Innervation Patterns of Serotonin-Immunoreactive Neurons in the Brain}

The low number of serotonin-immunoreactive neurons gives rise to many neuronal processes, spreading widely in the brain. Except the neurons in the cluster of PR-L, the identified neurons projected across the two brain hemispheres via commissures and innervated the contralateral brain hemisphere (Figures 4A,D). The bilateral serotonin-immunoreactive neurons form at least six commissures: (1) the posterior protocerebral commissure 

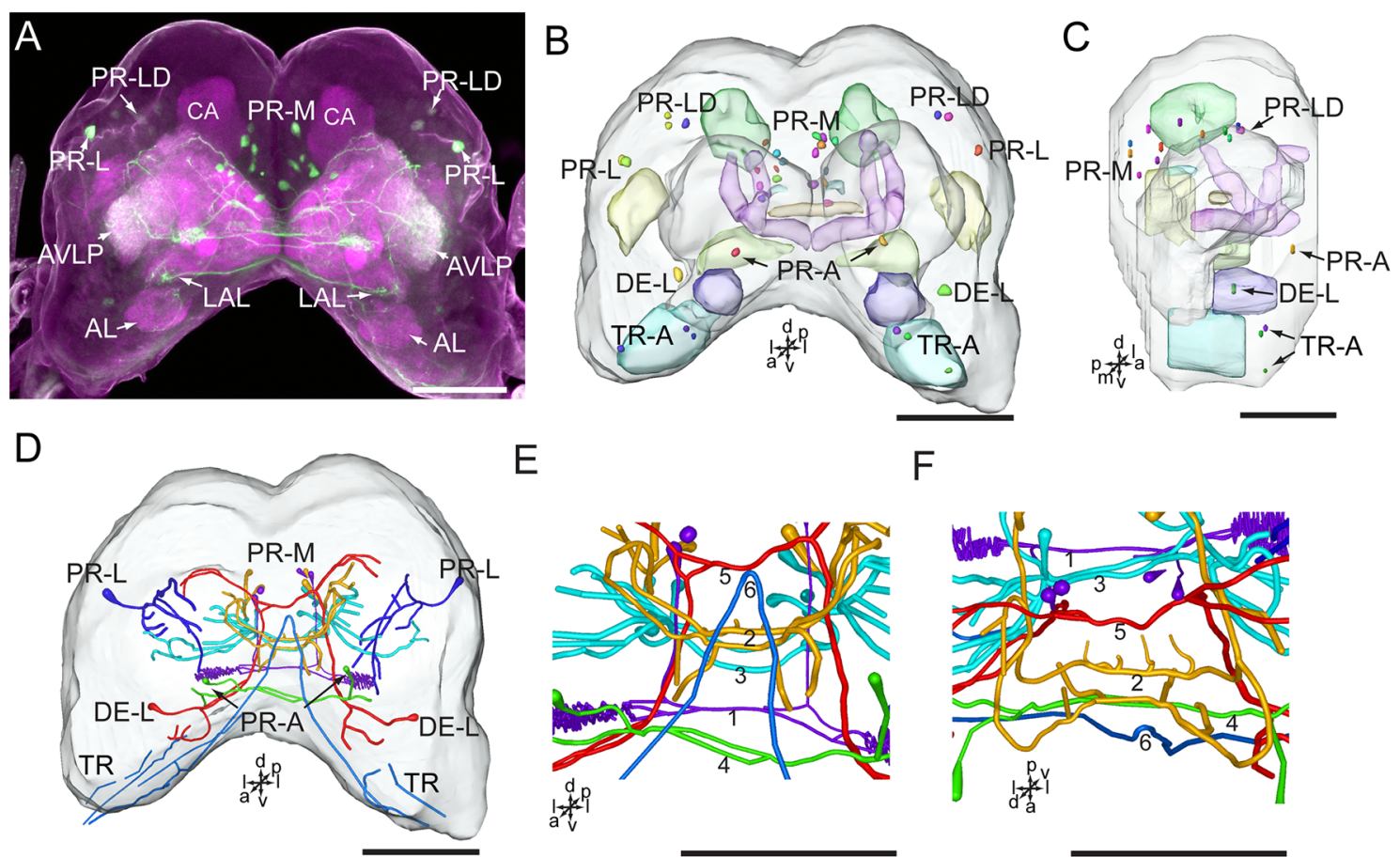

E

$\mathrm{F}$
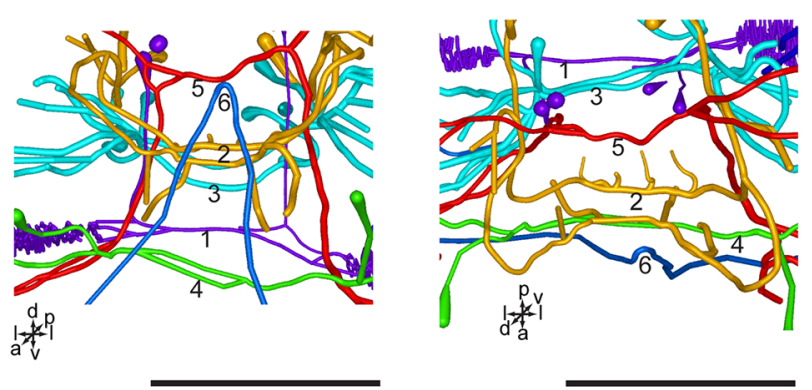

FIGURE 4 | Confocal image and three-dimensional reconstructions showing the distribution of the serotonin-immunoreactive neuronal processes and cell bodies in the brain. (A) Projection view of the confocal stack images. (B) Three-dimensional reconstructions of the brain including the labeled cell body clusters in frontal view. (C) Three-dimensional reconstructions of the brain including labeled cell body clusters in sagittal view. (D) Reconstructed skeleton trees of the thick neuronal processes showing their projection patterns in frontal view. (E) Reconstructed skeleton trees of 1-6 commissures in frontal view. (F) Reconstructed skeleton trees of 1-6 commissures in dorsal view. AL, antennal lobe; AVLP, anterior ventrolateral protocerebrum; CA, calyx; LAL, lateral accessory lobe; TR, tritocerebrum. DE-L, PR-A, PR-L, PR-LD, PR-M, and TR-A are cell clusters. 1, the posterior protocerebral commissure linking the POTU; 2, the median protocerebral commissure linking the CB and bilateral SIP; 3 , the posterior great commissure linking the bilateral AVLP and the CL; 4, the LAL commissure; 5 , the dorsal protocerebral commissure linking the bilateral AL and the SLP; 6 , the anterior protocerebral commissure linking the bilateral tritocerebrum. Directions: a, anterior; $d$, dorsal; l, lateral; $p$, posterior; v, ventral. Scale bars, $100 \mu \mathrm{m}$.

TABLE 1 | Number, location, and innervation area of serotonin-immunoreactive neurons in the brain and the gnathal ganglion of Helicoverpa armigera larvae.

\begin{tabular}{lllll}
\hline & Cell body cluster & Number of neurons $(\boldsymbol{n})$ & Location of cell body & Innervation areas \\
\hline Brain & PR-M & $17-20(7)$ & $\begin{array}{l}\text { Medial region of posterior protocerebrum, } \\
\text { ventrolateral to the calyx }\end{array}$ & CB, bilateral AVLP, CL, CRE, SIP, POTU, and LAL \\
& & $5-6(7)$ & Dorsolateral protocerebrum & Not resolved \\
& PR-LD & $2-3(7)$ & Lateral protocerebrum, dorsolateral to OL & Ipsilateral OL, PLP, PVLP, SLP, and LAL \\
PR-L & $2-4(7)$ & Anterior to protocerebrum, dorsal to AL & Bilateral LAL \\
PR-A & $2(7)$ & Lateral to AL & Contralateral AL and bilateral SLP \\
DE-L & $6(2)$ & Anteromedial tritocerebrum & Not resolved \\
TR-A & $3-4(7)$ & Anterior dorsal gnathal ganglion & Anterior mandibular neuromere and tritocerebrum \\
GNG & $2(7)$ & Anterior ventral gnathal ganglion & Not resolved \\
GNG-AD & $3-4(6)$ & Medial dorsal gnathal ganglion & Not resolved \\
GNG-AV & $4(7)$ & Anterior lateral gnathal ganglion & Mandibular neuromere and tritocerebrum \\
GNG-M & $4(7)$ & Medial lateral gnathal ganglion & Maxillary neuromere and tritocerebrum \\
GNG-L1 & $4(7)$ & Posterior lateral gnathal ganglion & Labial neuromere and tritocerebrum
\end{tabular}

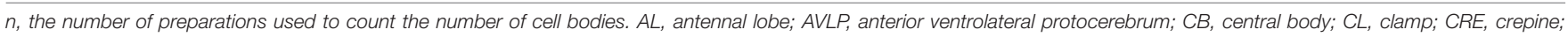
ILP, inferior lateral protocerebrum; LAL, lateral accessory lobe; OL, optic lobe; PLP, posterior lateral protocerebrum; POTU, posterior optic tubercle; PVLP, posterior ventrolateral protocerebrum; SIP, superior intermediate protocerebrum; SLP, superior lateral protocerebrum.

has processes to the POTU; (2) the median protocerebral commissure has processes to the SIP and the $\mathrm{CB}$; (3) the posterior great commissure has processes to the AVLP and the clamp; (4) the LAL commissure has processes to the LAL; (5) the dorsal protocerebral commissure has processes to the SLP and the AL; and (6) the anterior protocerebral commissure connects the tritocerebra of two brain hemispheres (Figures 3, 4E,F).

In accordance with the innervation terminal regions, the cells in the cluster of PR-M were classified as PR-M1, PR-M2, 


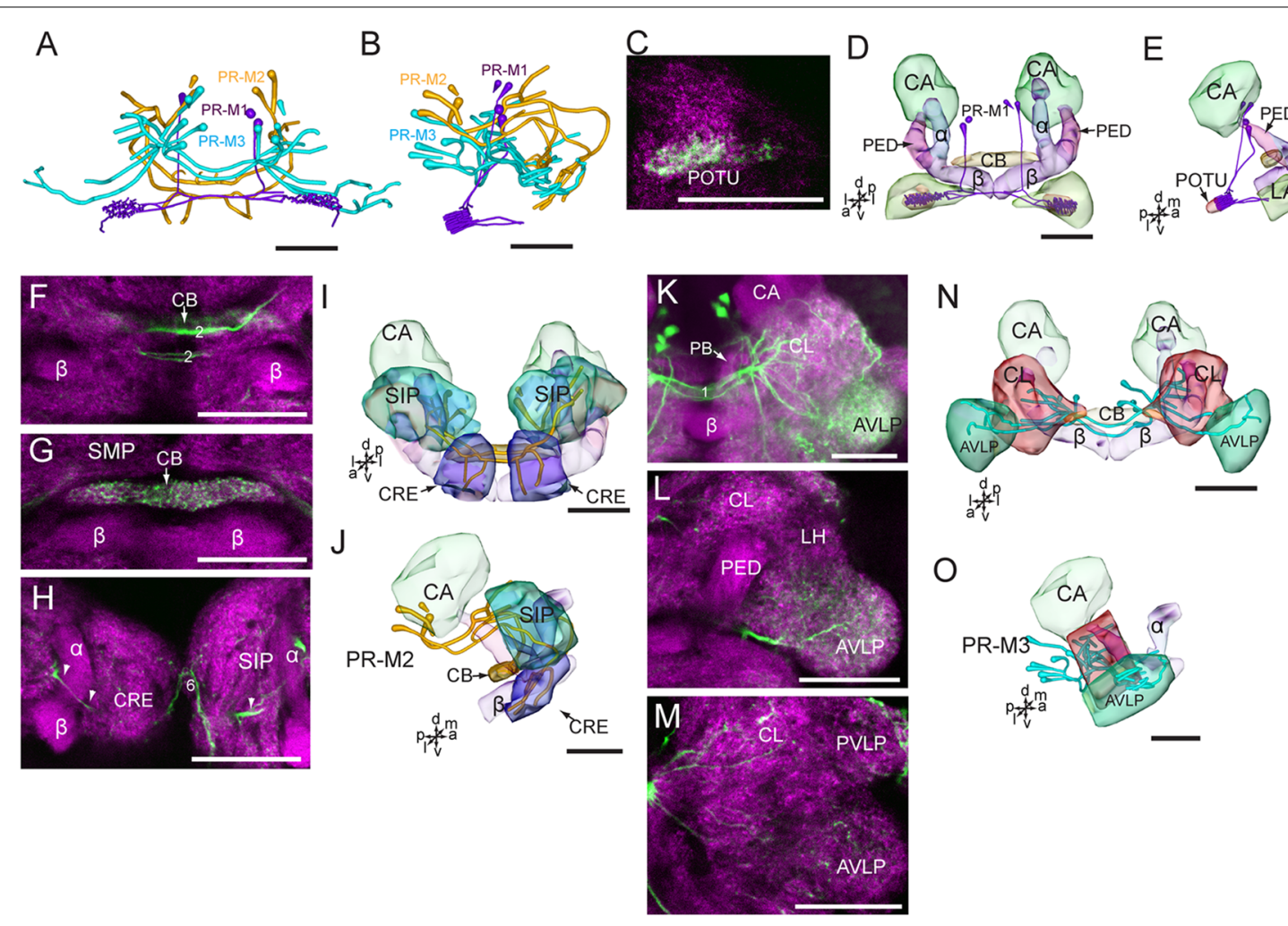

FIGURE 5 | Confocal images and three-dimensional reconstructions showing the distribution of the serotonin-immunoreactive neuronal processes and cell bodies in the protocerebrum. (A) Reconstructed skeleton trees of PR-M neurons in frontal view. (B) Reconstructed skeleton trees of PR-M neurons in dorsal view. (C) Confocal image showing the serotonin-immunoreactive processes (green) in the POTU. (D) Three-dimensional reconstructions of serotonin-immunoreactive neurons in the cluster of PR-M1 and related neuropils in frontal view. (E) Three-dimensional reconstructions of the serotonin-immunoreactive neurons of PR-M1 and related neuropils in lateral view. (F-H) Confocal images showing the serotonin-immunoreactive processes of neurons PR-M2 (green). (I) Three-dimensional reconstructions of the serotonin-immunoreactive neurons in the cluster of PR-M2 and related neuropils in frontal view. (J) Three-dimensional reconstructions of the serotonin-immunoreactive neurons in the cluster of PR-M2 and related neuropils in lateral view. (K-M) Confocal images showing the serotonin-immunoreactive processes of neurons PR-M3 (green). (N) Three-dimensional reconstructions of the serotonin-immunoreactive neurons in the cluster of PR-M3 and related neuropils in frontal view. (O) Three-dimensional reconstructions of the serotonin-immunoreactive neurons in the cluster of PR-M3 and related neuropils in lateral view. $\alpha$, alpha lobe; AVLP, anterior ventrolateral protocerebrum; $\beta$, belta lobe; CA, calyx; CB, central body; CL, clamp; CRE, crepine; LAL, lateral accessory lobe; PB, protocerebral bridge; PED, pedunculus; POTU, posterior optic tubercle; PVLP, posterior ventrolateral protocereburm; SIP, superior intermediate protocerebrum; SMP, superior medial protocerebrum. PR-M1-3 are cell clusters. 2, the median protocerebral commissure linking the CB and bilateral SIP; 6 , the anterior protocerebral commissure linking the bilateral tritocerebrum. Directions: a, anterior; $d$, dorsal; l, lateral; $\mathrm{m}$, medial; $\mathrm{p}$, posterior; $\mathrm{v}$, ventral. Scale bars, $50 \mu \mathrm{m}$.

and PR-M3. The cells of PR-M1, about four, located in the anterior part of the cluster of PR-M, have bilaterally symmetrical processes throughout the POTU (Figures 5A-E). A side branch projects further anteriorly from the POTU to the LAL (Figure 5E). The cells of PR-M2, about four, located in the medial part of the cluster of PR-M, have symmetrical processes in the SIP, the crepine, and the CB (Figures 5F-J). Two thick neurites originating in these cells run parallelly and form the medial protocerebral commissure nearby the $\mathrm{CB}$ (Figure 5F). One neurite gives rise to fine processes in $\mathrm{CB}$, and is uniform throughout (Figure 5G). One neurite runs bypass the $\alpha$ lobe and gives rise to arborizations in the crepine (Figure $\mathbf{5 H}$ ). The cells of PR-M3, about 12, located in the posterior part of the cluster of PR-M, have bilaterally symmetrical processes throughout the AVLP and the clamp (Figures 5K-O). The thick neurites originating from these cells run parallelly and form the posterior great commissure (Figure $\mathbf{5 A}$ ). It was difficult to identify a single neuron to trace the processes and examine the innervation pattern in the ipsilateral and contralateral brain regions.

The neurites of cells in the cluster of PR-L run medially and give rise to several branches projecting to different regions in the ipsilateral brain hemisphere, including the OL, the LAL, the SLP, the PVLP, and the posterior lateral protocerebrum (Figures 6A-E). Neurites from the cells in the cluster of PR-A form the LAL commissure and project to the anterior and ventral portions of the LAL (Figures 6F-H).

The two cells in the cluster of DE-L are deutocerebral neurons, they project dorsoposteriorly into the ipsilateral protocerebrum via the medial $\mathrm{AL}$ tract, crossed the midline dorsal to the $\mathrm{CB}$, and extend to the contralateral $\mathrm{AL}$, where innervated the entire $\mathrm{AL}$ (Figures 7A-F). The cells also extended some fine branches in the bilateral SLP, ventral to the calyx of the MB (Figure 7D).

The cells in the cluster of PR-LD, and TR-A were weakly stained, and we were not able to detect neuronal processes from 

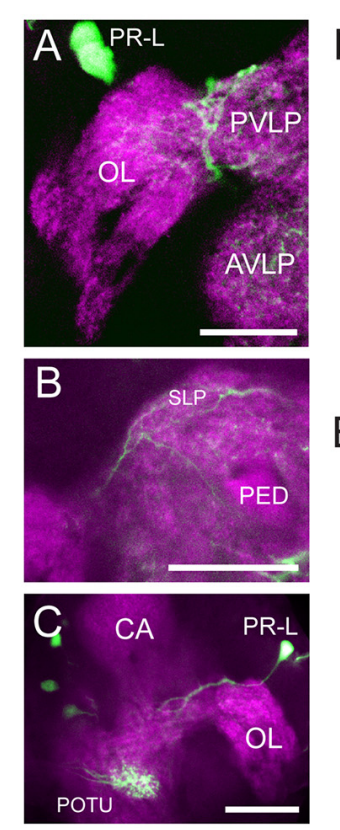

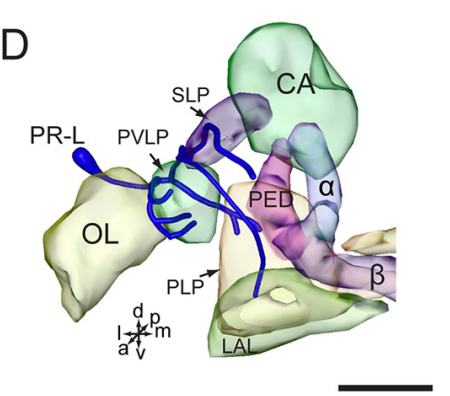

E

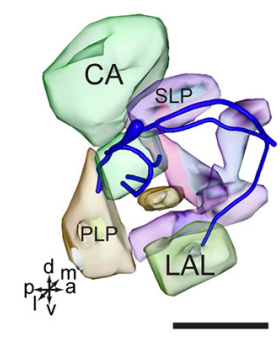

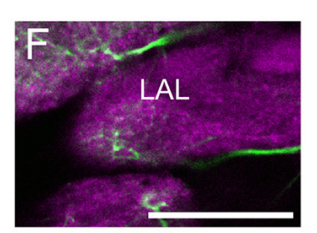

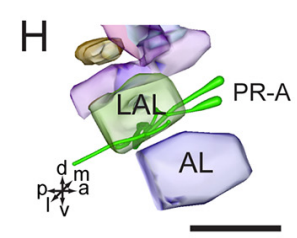

G

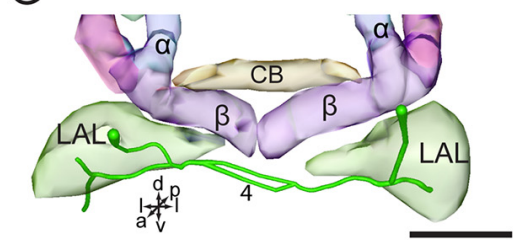

FIGURE 6 | Confocal images and three-dimensional reconstructions showing the distribution of the serotonin-immunoreactive neuronal processes and cell bodies in the cluster of PR-L and PR-A in the protocerebrum. (A-C) Confocal images showing the serotonin-immunoreactive processes of neurons PR-L (green).

(D) Three-dimensional reconstructions of the serotonin-immunoreactive neurons in the cluster of PR- $L$ and related neuropils in frontal view. (E) Three-dimensional reconstructions of the serotonin-immunoreactive neurons in the cluster of PR- $L$ and related neuropils in lateral view. (F) Confocal image showing the serotonin-immunoreactive processes of neurons PR-A (green). (G) Three-dimensional reconstructions of the serotonin-immunoreactive neurons in the cluster of PR-A and related neuropils in frontal view. $\mathbf{( H )}$ Three-dimensional reconstructions of the serotonin-immunoreactive neurons in the cluster of PR-A and related neuropils in lateral view. $\alpha$, alpha lobe; AL, antennal lobe; AVLP, anterior ventrolateral protocerebrum; $\beta$, belta lobe; CA, calyx; CB, central body; LAL, lateral accessory lobe; PED, pedunculus; PLP, posterior lateral protocerebrum; POTU, posterior optic tubercle; PVLP, posterior ventrolateral protocereburm; SLP, superior lateral protocerebrum. PR-A and PR-L are cell clusters. 4, the LAL commissure. Directions: a, anterior; d, dorsal; I, lateral; m, medial; p, posterior; v, ventral. Scale bars, 50 $\mu$ m.

these cells. In the tritocerebrum, we observed some serotoninimmunoreactive neuronal processes, which may originate in the frontal ganglion and the gnathal ganglion (Figures $7 \mathbf{G}-\mathbf{K}, \mathbf{8}$ ). In the frontal ganglion, there is a single big serotoninimmunoreactive neuron, and some neurites connect to the tritocerebrum through the frontal connectives (Figure 7I).

The wide-field serotonin-immunoreactive neurons spread fine processes throughout the brain, however, some regions of neuropils lacked serotonin, including the calyx, the pedunculus, $\alpha$ lobe, $\beta$ lobe, the $\mathrm{PB}$, the lateral horn, and the medial and dorsal portions of the LAL (Figures 5F,G,K,L, 6B,C,F, 7D).

\section{Serotonin-Immunoreactivity in the Gnathal Ganglion of $\boldsymbol{H}$. armigera larvae}

There are about 20-22 serotonin-immunoreactive neurons in the gnathal ganglion, distributed in several clusters; each cluster contains 2-4 neurons (Table 1; Figure 8). The cluster of GNG-AD contains 3-4 cell bodies, located in the medial region of the anteriodorsal gnathal ganglion. The cluster of GNG-AV contains two neurons and GNG-M contains 3-4 neurons, located in the anterior and medial regions of ventral gnathal ganglion. The cells in these clusters were weakly stained, and no neurites extending from their cell bodies were detected. In each lateral side of the gnathal ganglion, there are three clusters, GNG-L1-GNG-L3; each cluster contains two neurons.
The cells in the cluster of GNG-L1 are situated in the dorsal lateral gnathal ganglion, whereas GNG-L2 and GNG-L3 cells are located ventrolaterally.

Three cell clusters in the lateral gnathal ganglion, GNGL1-GNG-L3, were associated with three neuromeres, respectively, i.e., mandibular neuromere, maxillary neuromere, and labial neuromere (Figures 8A-D,G,H). From two cells of one cluster on each side of the ganglion, major processes extend into the contralateral hemisphere via a commissure, which contained four neurites. In the mandibular neuromere, at least two branches extended from the cell; one laterally and the other medially. Both branches projected ventroanteriorly to the tritocerebrum through circumoesophageal connectives and had many fine arborizations in the medial regions of each gnathal neuromere hemisphere and tritocerebrum (Figures 7G,H, 8A-D,G,H). In the maxillary and labial neuromeres, the serotonin-immunoreactive neuron processes show a branching pattern similar to that of the mandibular neuromere. The branches run across the midline via the commissure and project through the proceeding neuromere, and terminate in the tritocerebrum. In addition to the prominent commissures in the ventral part of the maxillary and labial neuromeres, a dorsal immunoreactive commissure was observed at the dorsal surface of each neuromere (indicated by arrows in Figures 8C,G). 

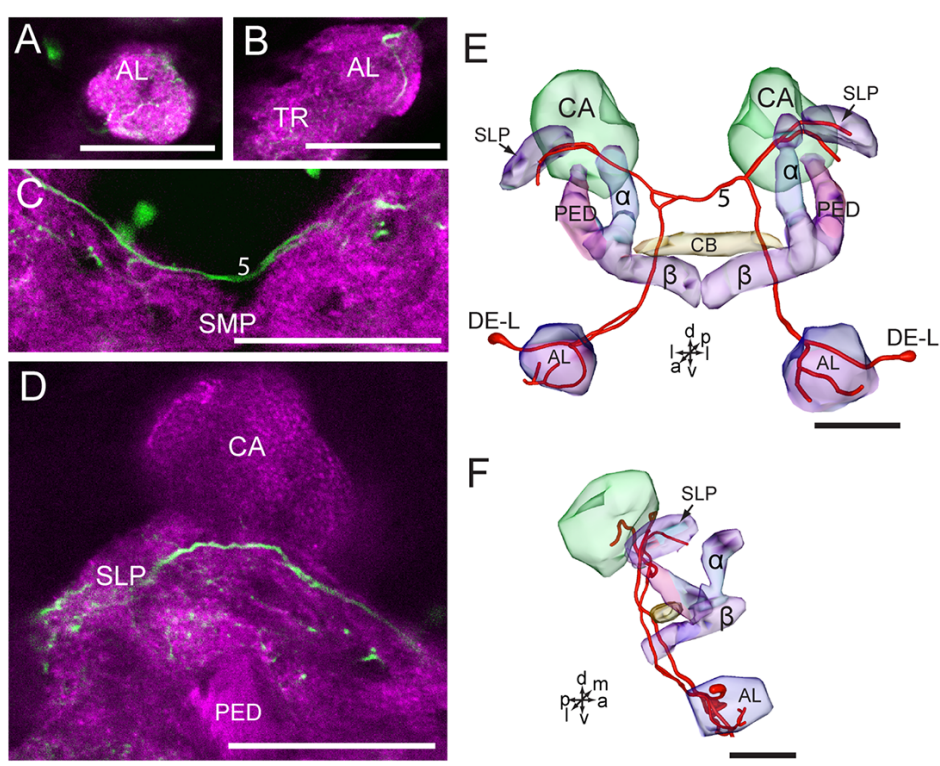
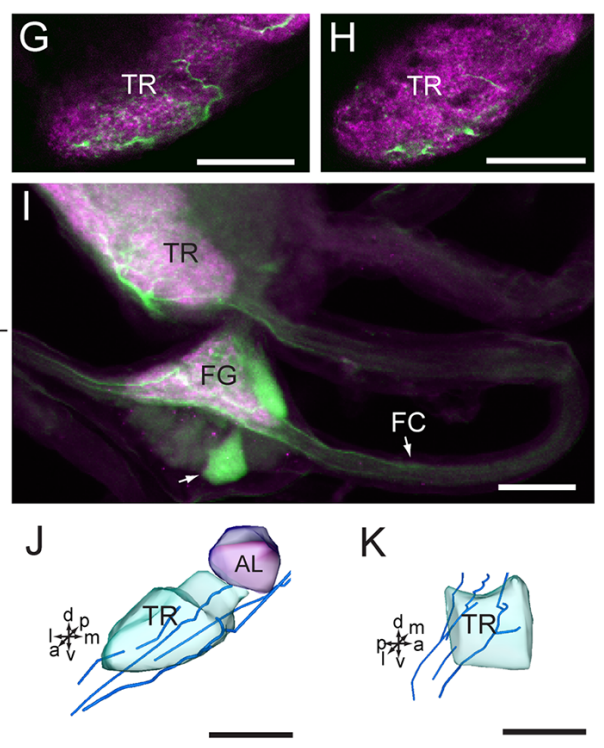

K

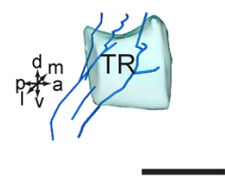

FIGURE 7 | Confocal images and three-dimensional reconstructions showing the distribution of serotonin-immunoreactive neuronal processes in the protocerebrum, deutocerebrum and tritocerebrum. (A-D) Confocal images showing the serotonin-immunoreactive processes of neurons DE-L (green). (E) Three-dimensional reconstructions of the serotonin-immunoreactive neurons in the cluster of DE- $L$ and related neuropils in frontal view. (F) Three-dimensional reconstructions of the serotonin-immunoreactive neurons in the cluster of DE-L and related neuropils in lateral view. (G,H) Confocal images showing the serotonin-immunoreactive processes in the tritocerebrum (green). (I) Confocal image showing the serotonin-immunoreactive processes in the tritocerebrum and the frontral ganglion (green). (J) Three-dimensional reconstructions of the serotonin-immunoreactive processes in the tritocerebrum and related neuropils in frontal view. (K) Three-dimensional reconstructions of serotonin-immunoreactive processes in the triotocerebrum in lateral view. $\alpha$, alpha lobe; AL, antennal lobe; $\beta$, belta lobe; CA, calyx; FC, frontal connective; FG. Frontal ganglion; PED, pedunculus; SLP, superior lateral protocerebrum; SMP, superior medial protocerebrum; TR, tritocerebrum. DE-L are cell bodies. 5, the dorsal protocerebral commissure linking the bilateral AL and the SLP. Directions: a, anterior; d, dorsal; I, lateral; m, medial; $\mathrm{p}$, posterior; v, ventral. Scale bars, $50 \mu \mathrm{m}$.

From each cell in the cluster of GNG-AD, at least one neurite projects to the tritocerebrum, exhibiting many fine immunoreactive arborizations in the anterior part of mandibular neuromere (Figures $\mathbf{8 A}, \mathbf{B}, \mathbf{G}, \mathbf{H}$ ). We were able not to detect serotonin-immunoreactive processes originating in the weakly stained cells in the cluster of GNG-AV and GNG-M (Table 1).

\section{DISCUSSION}

\section{Number of Serotonin-Immunoreactive Neurons in the Brain}

For the first time, the serotonergic neurons in the brain and the gnathal ganglion of $H$. armigera larvae were comprehensively revealed by performing immunohistochemistry with anti-serotonin serum. There are about 40 serotoninimmunoreactive neurons in the brain and about 20 in the gnathal ganglion of $H$. armigera larvae. The numbers are similar to those reported in larvae of the sphinx moth, Manduca sexta, flies D. melanogaster, Calliphora erythrocephala and Sarcophaga bullata, and the beetle Tenebrio molitor (Table 2, Nässel and Cantera, 1985; Vallés and White, 1988; Granger et al., 1989; Griss, 1989; Breidbach, 1990; Huser et al., 2012). Compared to the large number of neurons in the brain and the gnathal ganglion, the number of serotonin-immunoreactive neurons is low. In adults of $M$. sexta and D. melanogaster, the number of serotonin-immunoreactive cell bodies in the gnathal ganglion is also about 20 (Table 2, Vallés and White, 1988; Homberg and Hildebrand, 1989a; Sitaraman et al., 2008), suggesting that the serotonin-immunoreactive neurons in larvae persist during metamorphosis to adults. Similarly, in the central brain of adults of these species, e.g., brain neuropils excluding the $\mathrm{OL}$, the number of serotonin-immunoreactive cell bodies is about 40 (Vallés and White, 1988; Homberg and Hildebrand, 1989a; Sitaraman et al., 2008). Similar number were found in the honeybee Apis mellifera, the wasp Trichogramma evanescens, and the blood-feeding bug Rhodnius prolixus, which are lower than the number of 200 in the cockroach Periplaneta americana (Table 2, Klemm et al., 1984; Schürmann and Klemm, 1984; Lange et al., 1988; van der Woude and Smid, 2017). The OL in larvae (the larval optic center) is an OL anlage that develops during metamorphosis to the adult form with the neuropils of lamina, medulla, and lobula complex (Nässel et al., 1987; Breidbach, 1990; Seidel and Bicker, 1996; Tang et al., 2014). The differentiation of serotonin-immunoreactive neurons in the OL is dependent on the development of OL neuropils (Nässel et al., 1987). Most serotonin-immunoreactive cell bodies in the OL are local amacrine neurons that innervate the local neuropils of the OL (Homberg and Hildebrand, 1989b). There are about 600 serotonin-immunoreactive cell bodies located in the OL of M. sexta and 20 in D. melanogaster (Homberg and Hildebrand, 1989b; Sitaraman et al., 2008). For comparison, it 


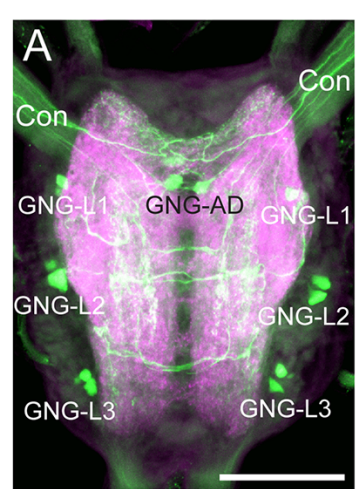

E

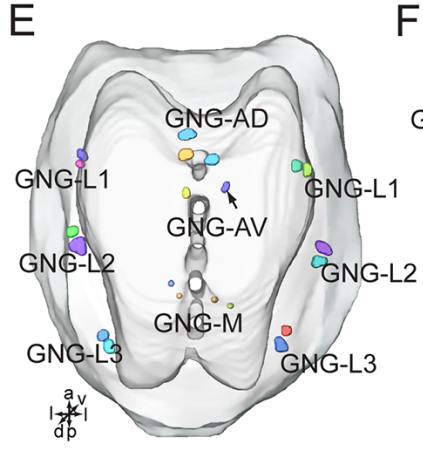

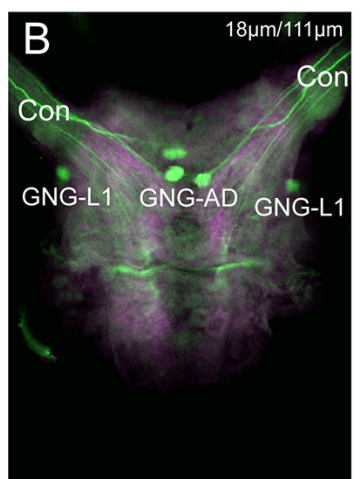

$\mathrm{F}$

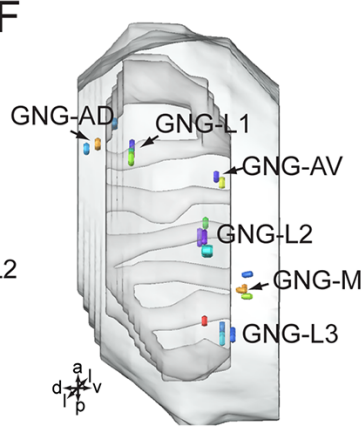

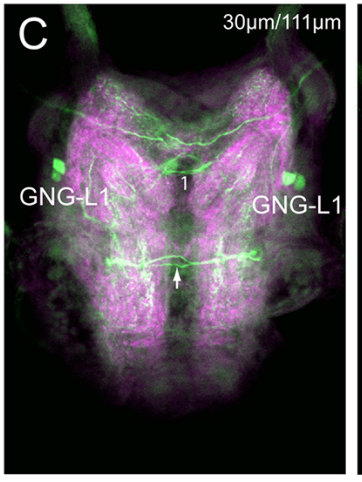

G

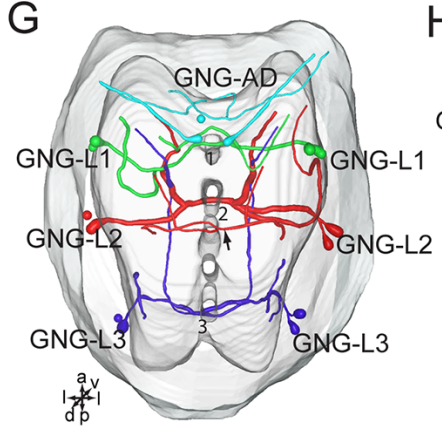

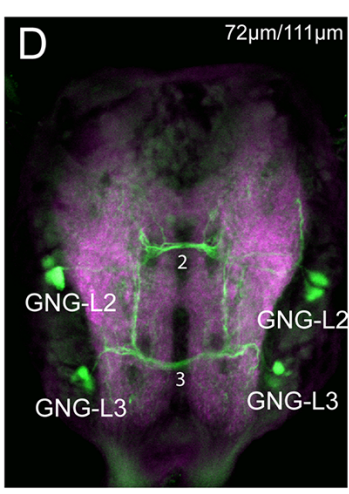

$\mathrm{H}$

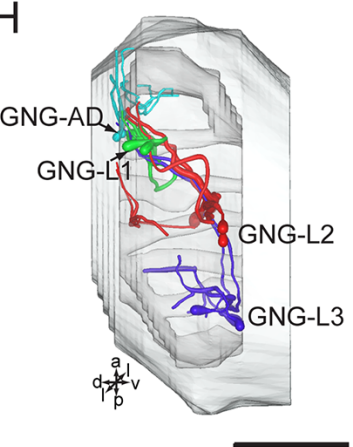

FIGURE 8 | Confocal images and three-dimensional reconstructions showing the distribution of the serotonin-immunoreactive neuronal processes and cell bodies in the gnathal ganglion. (A) Projection view of confocal stack images of the gnathal ganglion. Serotonin-immunoreactive neurons in green and the surrounding neuropil in magenta. (B-D) Serial confocal images of the gnathal ganglion at different depths. (E) Three-dimensional reconstructions of the gnathal ganglion including labeled cell body clusters in frontal view. Arrow in (C) indicated the dorsal commissure formed by neurons of GNG-L2. (F) Three-dimensional reconstructions of the gnathal ganglion including the labeled cell body clusters in lateral view. (G) Reconstructed skeleton trees of the thick neuronal processes showing their projection patterns in frontal view. Arrow indicates the dorsal commissure formed by neurons of GNG-L2. (H) Reconstructed skeleton trees of the thick neuronal processes showing their projection patterns in lateral view. Con, connective; GNG-L1-L3, GNG-AD, GNG-AV, and GNG-M are cell clusters. 1, the commissure formed by neurons of GNG1; 2, the commissure formed by neurons of GNG-L2; 3, the commissure formed by neurons of GNG-L3. Directions: a, anterior; d, dorsal; I, lateral; p, posterior; v, ventral. Scale bars, $100 \mu \mathrm{m}$.

is essential to examine the serotonin-immunoreactive neurons in adult $H$. armigera further. The similarity and difference in serotonin-immunoreactive neurons between the larvae and the adults could provide insight on the development and function of serotonin in different stages.

\section{Serotonin-Immunoreactive Neurons Associated With the Protocerebrum}

The location of serotonin-immunoreactive cell bodies and their branching patterns of $H$. armigera larvae are also similar to that of other studied insects (Vallés and White, 1988; Granger et al., 1989; Griss, 1989; Breidbach, 1990). Most serotoninimmunoreactive neurons are wide-field neurons and have processes extending throughout the neuropils in the brain. Although the morphology of these neurons is very similar, their terminals in part invade some different areas among different species, suggesting species-specific modifications of arborization patterns.

In the protocerebrum, the cells in the cluster of PR-M have processes projecting to the $\mathrm{CB}$, and bilateral crepine, clamp, SIP, AVLP, and POTU. The arborizations in the CB, the clamp, the AVLP, and the POTU are quite dense. The cells in the cluster of
PR-L have processes projecting mainly to the ipsilateral posterior ventrolateral, posterior lateral, and SLP. A few processes of these cells reach the inner part of the ipsilateral OL. The cells in the cluster of PR-A have processes and project bilaterally to the LAL. The pattern of serotonin-immunoreactive arborizations in the superior and lateral protocerebrum is roughly similar between species. Comparisons of these neuropils between different species are difficult to make due to the scarcity of information. Therefore, comparisons of the serotonin-immuonstaining between $H$. armigera and other species are mainly focused on the prominent neuropils, the $\mathrm{MB}, \mathrm{CB}$, the $\mathrm{PB}$, the $\mathrm{LAL}$, and the POTU. The strong serotonin-immunoreactivity in the POTU in larvae, however, was first found in $H$. armigera. Previously, the serotonin-immunoreactive processes in the POTU were found in adults of $M$. sexta and the locust Schistocerca gregaria, but the staining intensity was lower (Homberg and Hildebrand, 1989a; Homberg, 1991; Beetz et al., 2015). Serotoninimmunoreactive neurons linking the LAL are similar in several insect species, i.e., D. meloanogaster, M. sexta, and T. molitor, dung beetles Scarabaeus lamarchki, S. satyrus, S. gragaria, and the aphid Acyrthosiphon pisum (Vallés and White, 1988; Granger et al., 1989; Homberg and Hildebrand, 1989a; 
TABLE 2 | Number of serotonin-immunoreactive neurons in the brain of different insect species.

\begin{tabular}{|c|c|c|c|c|}
\hline & Species & Central brain & Gnathal ganglion & Reference \\
\hline \multicolumn{5}{|l|}{ Larva } \\
\hline Beetle & Tenebrio molitor & 42 & - & Breidbach (1990) \\
\hline Moth & Manduca sexta & $36-40$ & 20 & Griss (1989) and Granger et al. (1989) \\
\hline \multirow[t]{3}{*}{ Flies } & Drosophila melanogaster & 40 & 16 & Vallés and White (1988) \\
\hline & Calliphora erythrocephala & 30 & 22 & Nässel and Cantera (1985) \\
\hline & Sarcophaga bullata & 32 & 22 & Nässel and Cantera (1985) \\
\hline \multicolumn{5}{|l|}{ Adult } \\
\hline Cockroach & Periplaneta americana & 200 & - & Klemm et al. (1984) \\
\hline Bug & Rhodnius prolixus & 40 & 13 & Lange et al. (1988) \\
\hline Beetle & Tenebrio molitor & 42 & - & Breidbach (1990) \\
\hline Wasp & Trichogramma evanescens & 36 & 22 & van der Woude and Smid (2017) \\
\hline Bee & Apis mellifera & 50 & - & Schürmann and Klemm (1984) \\
\hline Moth & Manduca sexta & 40 & 20 & Homberg and Hildebrand (1989a) \\
\hline Fly & Drosophila melanogaster & $38-40$ & $16-18$ & Vallés and White (1988), Sitaraman et al. (2008) and Huser et al. (2012) \\
\hline
\end{tabular}

"-", Not counted.

Breidbach, 1990; Homberg, 1991; Kollmann et al., 2011; Immonen et al., 2017). The presence of serotoninimmunoreactive processes in the $\mathrm{CB}$ also seems to be common across insect species. However, in $S$. gregaria and $P$. americana, the lower part of the $\mathrm{CB}$ is devoid of serotonin-immunoreactive processes (Klemm et al., 1984; Tyrer et al., 1984). The PB, which is associated with the $\mathrm{CB}$, as a part of the central complex, showed no serotoninimmunoreactivity in $H$. armigera larvae. The $\mathrm{PB}$ of $M$. sexta larvae and adults, as well as the wasp T. evanescens, the ant Harpegnathos saltator, and A. mellifera, also lacks serotoninimmunoreactive processes (Schürmann and Klemm, 1984; Granger et al., 1989; Homberg and Hildebrand, 1989a; Hoyer et al., 2005; van der Woude and Smid, 2017). In contrast, the $\mathrm{PB}$ of $S$. gregaria and $P$. americana contains dense serotonin-immunoreactive processes (Klemm et al., 1984; Tyrer et al., 1984). The prominent neuropils of the $\mathrm{MB}$, including the calyx, the pedunculus, and the $\alpha$ and $\beta$ lobes, lack serotonin-immunoreactivity in $H$. armigera larvae. Similar findings have been reported in larvae of $M$. sexta and D. melanogaster (Granger et al., 1989; Huser et al., 2012). In contrast, the MB of adult M. sexta and D. melanogaster contains fine serotonin-immunoreactive processes (Homberg and Hildebrand, 1989a; Sitaraman et al., 2008). These results suggest that some immunoreactive neurons are remodeled during the metamorphosis from larva to adult in moths. In general, the intrinsic neurons of the $\mathrm{MB}$, the Kenyon Cells, are devoid of serotonin across insect taxa, whereas extrinsic neurons of the $\mathrm{MB}$ show the considerably varied innervation patterns. For instance, the calyx of $A$. mellifera, the calyx collar of $H$. saltator, the calyx and the pedunculus of S. gregaria and Locusta migratoria, the inner part of the calyx and the upper part of the pedunculus of $P$. americana, and the pedunculus and the $\alpha$ and $\beta$ lobes of $T$. infestans contain no serotonin-immunoreactive processes (Klemm et al., 1984; Schürmann and Klemm, 1984; Tyrer et al., 1984; Ignell, 2001; Settembrini and Villar, 2004; Hoyer et al., 2005). In addition, in the larva of $H$. armigera, the lateral horn, an area associated with the $\mathrm{MB}$, lacks serotonin-immunoreactivity. To date, such findings have not been reported in other species.

\section{Serotonin-Immunoreactive Neurons Associated With Deutocerebrum}

A pair of deutocerebral serotonin-immunoreactive DE-L neurons were first reported in larva of M. sexta (Kent et al., 1987). These deutocerebral neurons have arborizations in the contralateral AL and bilateral SLP. The AL in the larval brain is also called the larval antennal center. In adults, the branching pattern persists and expands in the AL with the formation of glomeruli (Kent et al., 1987). Ultrastructural studies on the synaptic terminals indicated that the deutocerebral neuron is a feedback neuron to the AL for olfactory processing (Sun et al., 1993). Electrophysiological recordings demonstrated that the deutocerebral serotonin-immunoreactive neuron showing responses to odorants and mechanical stimuli (Hill et al., 2002; Zhao and Berg, 2009). The serotonin-immunoreactive deutocerebral neurons were found in all studied species but varied in the number of cells and innervation patterns. There is a single cell body in each $\mathrm{AL}$ in the species of Lepidoptera, Trichoptera, Diptera, Coleoptera, and Neuroptera and 2-8 cells in each AL in Orthoptera and Blattaria (Dacks et al., 2006). In Hymenoptera, however, the immunoreactive cell body in $\mathrm{AL}$ is absent, and the processes in the AL originate in an ascending neuron. In Orthoptera and Blattaria, the processes of the deutocerebral neurons innervate the ipsilateral hemisphere of the AL and the protocerebrum (Dacks et al., 2006).

\section{Serotonin-Immunoreactive Neurons Associated With Tritocerebrum}

The tritocerebrum is a largely reduced neuropil, which is hard to discriminate from the surrounding neuropils in the brain of many holometabolous insect species (Ito et al., 2014). In the larvae of $H$. armigera, however, the tritocerebrum is a distinct and large neuropil, which is similar to that of hemimetabolous species, such as the locust S. gregaria and the bug Apolygus lucorum (Kurylas et al., 2008; Tang et al., 2014; Xie et al., 2016). The cells in the cluster of TR-A in tritocerebrum were weakly stained with anti-serotonin serum and their neuronal processes were not detected. Throughout the tritocerebrum, however, serotonin-immunoreactive neuronal processes are abundant, and they may have multiple origins, 
including the frontal ganglion and the gnathal ganglion. A commissure in the front of the medial protocerebrarum formed by neurons which links the tritocerebrum, giving rise to some arborizations in the vicinity. Although the number of studies in other species is low, the serotonin-immunoreactivity in the tritocerebrum seems to be common across insect taxa (Nässel, 1988; Granger et al., 1989; Wegerhoff, 1999). The serotonin-immunoreactive neuronal processes in the tritocerebrum connect the protocerebrum, deutocerebrum, gnathal ganglion, and stomatogastric nervous system.

\section{Serotonin-Immunoreactive Neurons Associated With Gnathal Ganglion}

In the gnathal ganglion, the serotonin-immunoreactive neurons also show high conservation across insect taxa. All three neuromeres of the gnathal ganglion in $H$. armigera larvae, i.e., the mandibular, maxillary, and labial neuromeres contain widespread processes originating in serotonin-immunoreactive cell clusters on both sides. The thick processes form a horseshoe pattern, cross the midline via a commissure and project anteriorly to the contralateral tritocerebrum. Such neurons and their branching patterns were also found in larvae of M. sexta, T. molitor and the flies, D. melanogaster, C. erythrocephala, S. bullata (Nässel and Cantera, 1985; Griss, 1989; Breidbach, 1990; Huser et al., 2012). In adults of these species, serotoninimmunoreactive neurons and processes in the gnathal ganglion well persist during metamorphosis. Similar neurons were also found in S. gregaria, A. mellifera, and P. americana (Bishop and O'Shea, 1983; Tyrer et al., 1984; Rehder et al., 1987; Vallés and White, 1988; Griss, 1989; Homberg and Hildebrand, 1989a; Breidbach, 1990). Each cell cluster contains two cell bodies. In flies, however, there are $2-5$ cell bodies in each cluster (Nässel and Cantera, 1985; Vallés and White, 1988; Huser et al., 2012). In addition, there are 3-4 large serotoninimmunoreactive cells the medial gnathal ganglion. Similar results were also found in S. gregaria, $P$. americana, and larval and adult M. sexta (Bishop and O'Shea, 1983; Tyrer et al., 1984; Griss, 1989; Homberg and Hildebrand, 1989a). They were identified as efferent neurons in the mandibular neuromere (Tyrer et al., 1984; Griss, 1989; Homberg and Hildebrand, 1989a). Intracellular recordings from such neurons of $M$. sexta larvae revealed overshooting soma spikes of large amplitude and long duration, which suggest these neurons are neurosecretory cells (Griss, 1989). In addition, in the gnathal ganglion of S. gregaria, four serotonin-immunoreactive neurons innervate the salivary gland (Tyrer et al., 1984); these neurons have not been found in H. armigera larvae.

In $H$. armigera larvae, compared to the brain, the neuropil volume of the gnathal ganglion is smaller and the sub-regions are fewer (Tang et al., 2014). Correspondingly, the number of serotonin-immunoreactive neurons in the gnathal ganglion is lower and neuronal branch patterns are more concise.

\section{CONCLUSION}

We have provided, for the first time, a comprehensive description of the serotonergic neuronal network in $H$. armigera larvae. In accordance with its widespread presence in insects, serotonin plays a variety of roles. In the present study, we found there are about 40 serotonin-immunoreactive neurons in the brain and about 20 in the gnathal ganglion. Most of these neurons are wide-field neurons giving rise to processes throughout the neuropils of the brain and the gnathal ganglion. In the central brain, serotonin-immunoreactive processes are present bilaterally in the tritocerebrum, the deutocerebrum, and major regions of the procerebrum, including the $C B$, the $L A L$, the clamp, the cripine, the superior protocerebrum, and the lateral protocerebrum. These results indicate that serotonin may play a variety of roles in $H$. armigera. Particularly, the AVLP, CB, and the POTU contain extensive serotonin-immunoreactive process terminals. The $\mathrm{CB}$ has been demonstrated to be a locomotion and navigation center, and the POTU is involved in navigation in locust (Pfeiffer and Homberg, 2014; Beetz et al., 2015). The AVLP in moths is a region involved in sound information processing (Pfuhl et al., 2014). The high serotonin-immunoreactivity in the $\mathrm{CB}$, the POTU and the AVLP might indicate that serotonin plays important roles in $H$. armigera larvae for locomotion and sound reception. However, the $\mathrm{MB}$, the lateral horn, and the $\mathrm{PB}$ are devoid of serotonin-immunoreactivity. The MB and the lateral horn are higher olfactory centers of insects. The absence of serotonin in these centers suggests that serotonin is not involved in the modulation of higher centers. Instead, serotonin has been demonstrated to modulate the olfactory information in the $\mathrm{AL}$ by giving the feedback to the protocerebrum (Kloppenburg and Mercer, 2008). In addition, the MB is the learning and memory center and serotonin has the function of learning and memory (Sitaraman et al., 2008, 2012). The absence of serotonin in the $\mathrm{MB}$ indicates serotonin may play no role in learning and memory in $H$. armigera larvae. In the gnathal ganglion, the serotonin-immunoreactive processes are also widespread, and most, if not all, of the neurons project to the tritocerebrum. The gnathal ganglion is the gustatory center, while the tritocerebrum is the stomatogastric center. The links between serotoninimmunoreactive neurons from these two centers suggest the serotonin plays important roles in feeding, from selection to intake digestion.

In summary, the results of the present study provide a comprehensive description of the serotonergic neuronal network in $H$. armigera larvae, and show a map of the neural architecture and the distribution of neural substances, allowing us to explore the neural mechanisms of behaviors, such as host selection, navigation, and feeding preference and plasticity, by using electrophysiological and pharmacological approaches on the target regions.

\section{DATA AVAILABILITY}

All datasets generated for this study are included in the manuscript.

\section{AUTHOR CONTRIBUTIONS}

Q-BT, W-BC and X-CZ: study concept, design and final manuscript. Q-BT, W-WS, Y-JC, G-YX and W-BC: acquisition 
of data. W-WS, G-YX, W-BC and X-CZ: analysis and interpretation of data. $\mathrm{W}-\mathrm{BC}$ and $\mathrm{X}-\mathrm{CZ}$ : drafting of the manuscript. Q-BT and X-CZ: obtained funding.

\section{FUNDING}

This work was supported by National Natural Science Foundation of China (31672367, U1604109); Program for

\section{REFERENCES}

Ali, D. W. (1997). The aminergic and peptidergic innervation of insect salivary glands. J. Exp. Biol. 200, 1941-1949.

Andrés, M., Seifert, M., Spalthoff, C., Warren, B., Weiss, L., Giraldo, D., et al. (2016). Auditory efferent system modulates mosquito hearing. Curr. Biol. 26, 2028-2036. doi: 10.1016/j.cub.2016.05.077

Anstey, M. L., Rogers, S. M., Ott, S. R., Burrows, M., and Simpson, S. J. (2009). Serotonin mediates behavioral gregarization underlying swarm formation in desert locusts. Science 323, 627-630. doi: 10.1126/science.1165939

Beetz, M. J., el Jundi, B., Heinze, S., and Homberg, U. (2015). Topographic organization and possible function of the posterior optic tubercles in the brain of the desert locust Schistocerca gregaria. J. Comp. Neuol. 523, 1589-1607. doi: 10.1002/cne.23736

Bishop, C. A., and O'Shea, M. (1983). Serotonin immunoreactive neurons in the central nervous system of an insect (Periplaneta americana). J. Neurobiol. 14, 251-269. doi: 10.1002/neu.480140402

Boleli, I. C., and Paulino-Simões, Z. L. (1999). Mapping of serotoninimmunoreactive neurons of Anastrepha obliqua Macquart larvae. Revta. Bras. Zool. 16, 1099-1107. doi: 10.1590/s0101-81751999000400019

Breidbach, O. (1990). Serotonin-immunoreactive brain interneurons persist during metamorphosis of an insect: a development study of the brain of Tenebrio molitor L. (Coleoptera). Cell Tissue Res. 259, 345-360. doi: $10.1007 /$ bf00318458

Chen, B., Meinertzhagen, I. A., and Shaw, S. R. (1999). Circadian rhythms in light-evoked responses of the fly's compound eye and the effects of neuromodulators 5-HT and the peptide PDF. J. Comp. Physiol. A 185, 393-404. doi: 10.1007/s003590050400

Cymborowski, B. (1998). Serotonin modulates a photic response in circadian locomotor rhythmicity of adults of the blow fly Calliphora vicina. Physiol. Entomol. 23, 25-32. doi: 10.1046/j.1365-3032.1998.2310025.x

Dacks, A. M., Christensen, T. A., and Hildebrand, J. G. (2006). Phylogeny of a serotonin-immunoreactive neuron in the primary olfactory center of the insect brain. J. Comp. Neurol. 498, 727-746. doi: 10.1002/cne.21076

Dacks, A. M., Christensen, T. A., and Hildebrand, J. G. (2008). Modulation of olfactory information processing in the antennal lobe of Manduca sexta by serotonin. J. Neurophysiol. 99, 2077-2085. doi: 10.1152/jn.01372.2007

Ellen, C. W., and Mercer, A. R. (2012). Modulatory actions of dopamine and serotonin on insect antennal lobe neurons: insights from studies in vitro. J. Mol. Histol. 43, 401-404. doi: 10.1007/s10735-012-9401-7

Fernández, V. M., Giurfa, M., Devaud, J. M., and Farina, W. M. (2012). Latent inhibition in an insect: the role of aminergic signaling. Learn. Mem. 19, 593-597. doi: 10.1101/lm.028167.112

Fitt, G. P. (1989). The ecology of Heliothis species in relation to agroecosystems. Annu. Rev. Entomol. 34, 17-52. doi: 10.1146/annurev.ento.34.1.17

French, A. S., Simcock, K. L., Rolke, D., Gartside, S. E., Blenau, W., and Wright, G. A. (2014). The role of serotonin in feeding and gut contractions in the honeybee. J. Insect Physiol. 61, 8-15. doi: 10.1016/j.jinsphys.2013.12.005

Gatellier, L., Nagao, T., and Kanzaki, R. (2004). Serotonin modifies the sensitivity of the male silkmoth to pheromone. J. Exp. Biol. 207, 2487-2496. doi: 10.1242/jeb.01035

Giese, M., Gestrich, J., Massah, A., Peterle, J., Wei, H., and Stengl, M. (2018). GABA- and serotonin-expressing neurons take part in inhibitory as well as excitatory input pathways to circadian clock of the madeira cockroach Rhyparobia maderae. Eur. J. Neurosci. 47, 1067-1080. doi: 10.1111/ejn. 13863
Science and Technology Innovation Talents in Universities of Henan Province (19HASTIT011).

\section{ACKNOWLEDGMENTS}

We are grateful to $\mathrm{Ms}$ Juan $\mathrm{Qu}$ for insect rearing, Ms Meng-Li Yang for help with the laser scanning confocal microscopy, and Prof. Bente G. Berg (Norwegian University of Science and Technology) for help with the Amira software.

Glendinning, J. I., Domdom, S., and Long, E. (2001). Selective adaptation to noxious foods by a herbivorous insect. J. Exp. Biol. 204, 3355-3367.

Glendinning, J. I., Ensslen, S., Eisenberg, M. E., and Weiskopf, P. (1999). Diet-induced plasticity in the taste system of an insect: localization to a single transduction pathway in an identified taste cell. J. Exp. Biol. 202, 2091-2102.

Godenschwege, T. A., Reisch, D., Diegelmann, S., Eberle, K., Funk, N., Heisenberg, M., et al. (2004). Flies lacking all synapsins are unexpectedly healthy but are impaired in complex behaviour. Eur. J. Neurosci. 20, 611-622. doi: 10.1111/j.1460-9568.2004.03527.x

Granger, N. A., Homberg, U., Henderson, P., Towel, A., and Lauder, J. M. (1989). Serotonin-immunoreactive neurons in the brain of Manduca sexta during larval development and larval-pupal metamorphosis. Int. J. Dev. Neurosci. 7, 55-72. doi: 10.1016/0736-5748(89)90044-0

Griss, C. (1989). Serotonin-immunoreactive neurons in the suboesophageal ganglion of caterpillar of the hawk moth Manduca sexta. Cell Tissue Res. 258, 101-109. doi: 10.1007/bf00223149

Haselton, A. T., Downer, K. E., Zylstra, J., and Stoffolano, J. G. Jr. (2009). Serotonin inhibits protein feeding in the blow fly Phormia regina (Meigen). J. Insect Behav. 22, 452-463. doi: 10.1007/s10905-009-9184-1

Hill, E. S., Iwano, M., Gatellier, L., and Kanzaki, R. (2002). Morphology and physiology of the serotonin-immunoreactive putative antennal lobe feedback neuron in the male silkmoth Bombyx mori. Chem. Senses 27, 475-483. doi: 10.1093/chemse/27.5.475

Hinks, C. F. (1967). Relationship between serotonin and the circadian rhythm in some nocturnal moths. Nature 214, 386-387. doi: 10.1038/214386b0

Homberg, U. (1991). Neuroarchitecture of the central complex in the brain of the locust Schistocerca gregaria and S. americana as revealed by serotonin immunocytochemistry. J. Comp. Neurol. 303, 245-254. doi: 10.1002/cne. 903030207

Homberg, U., and Hildebrand, J. G. (1989a). Serotonin-immunoreactive neurons in the median protocerebrum and suboesophageal ganglion of the sphinx moth Manduca sexta. Cell Tissue Res. 258, 1-24. doi: 10.1007/bf00223139

Homberg, U., and Hildebrand, J. G. (1989b). Serotonin-immunoreactivity in the optic lobes of the sphinx moth Manduca sexta and colocalization with FMRFamide and $\mathrm{SCP}_{\mathrm{B}}$ immunoreactivity. J. Comp. Neurol. 288, 243-253. doi: $10.1002 /$ cne.902880204

Hoyer, S. C., Liebig, J., and Rössler, W. (2005). Biogenic amines in the ponerine ant Harpegnathos saltator: serotonin and dopamine immunoreactivity in the brain. Arthropod Struct. Dev. 34, 429-440. doi: 10.1016/j.asd.2005. 03.003

Huser, A., Rohwedder, A., Apostolopoulou, A. A., Widmann, A., Pfitzenmaier, J. E., Maiolo, E. M., et al. (2012). The serotonergic central nervous system of the Drosophila larva: anatomy and hehavioral function. PLoS One 7:e47518. doi: 10.1371/journal.pone.0047518

Ignell, R. (2001). Monoamines and neuropeptides in antennal lobe interneurons of the desert locust, Schistocerca gregaria: an immunocytochemical study. Cell Tissue Res. 306, 143-156. doi: 10.1007/s004410 100434

Immonen, E. V., Dacke, M., Heinze, S., and El Jundi, B. (2017). Anatomical organization of the brain of a diurnal and a nocturnal dung beetle. J. Comp. Neurol. 525, 1879-1908. doi: 10.1002/cne.24169

Ito, K., Shinomiya, K., Ito, M., Armstrong, J. D., Boyan, G., Hartenstein, V., et al. (2014). A systematic nomenclature for the insect brain. Neuron 81, 755-765. doi: 10.1016/j.neuron.2013.12.017 
Kaufmann, L., Schürmann, F., Yiallouros, M., Harrewijin, P., and Kayser, H. (2004). The serotonergic system is involved in feeding inhibition by pymetrozine. Comparative studies on a locust (Locusta migratoria) and an aphid (Myzus persicae). Comp. Biochem. Physiol. C Toxicol. Pharmacol. 138, 469-483. doi: 10.1016/j.cca.2004.08.007

Kent, K. S., Hoskins, S. G., and Hildebrand, J. G. (1987). A novel serotoninimmunoreactive neuron in the antennal lobe of the sphinx moth Manduca sexta persists throughout postembryonic life. J. Neurobiol. 18, 451-465. doi: 10.1002/neu.480180506

Klemm, N., Steinbusch, H. W. M., and Sundler, F. (1984). Distribution of serotonin-containing neurons and their pathways in the supraoesophageal ganglion of the cockroach Periplaneta americana (L.) as revealed by immunocytochemistry. J. Comp. Neurol. 225, 387-395. doi: 10.1002/cne. 902250306

Kloppenburg, P., and Mercer, A. R. (2008). Serotonin modulation of moth central olfactory neurons. Annu. Rev. Entomol. 53, 179-190. doi: 10.1146/annurev. ento.53.103106.093408

Kollmann, M., Minoli, S., Bonhomme, J., Homberg, U., Schachtner, J., Tagu, D., et al. (2011). Revisiting the antatomy of the central nervous system of a hemimetabolous model insect species: the pea aphid Acyrthosiphn pisum. Cell Tissue Res. 343, 343-355. doi: 10.1007/s00441-010-1099-9

Kurylas, A. E., Rohlfing, T., Krofczik, S., Jenett, A., and Homberg, U. (2008). Standardized atlas of the brain of the desert locust, Schistocerca gregaria. Cell Tissue Res. 333, 125-145. doi: 10.1007/s00441-008-0620-x

Lange, A. B., Orchard, I., and Lloyd, R. J. (1988). Immunohistochemical and electrochemical detection of serotonin in the nervous system of the bloodfeeding bug, Rhodnius prolixus. Arch. Insect Biochem. Physiol. 8, 187-201. doi: 10.1002/arch.940080305

Leitinger, G., Pabsta, M. A., and Kralb, K. (1999). Serotonin-immunoreactive neurones in the visual system of the praying mantis: an immunohistochemical, confocal laser scanning and electron microscopic study. Brain Res. 823, 11-23. doi: 10.1016/s0006-8993(98)01339-0

Linn, C. E. Jr., and Roelofs, W. L. (1986). Modulatory effects of octopamine and serotonin on male sensitivity and periodicity of response to sex pheromone in the cabbage looper moth, Trichoplusia ni. Arch. Insect Biochem. Physiol. 3, 161-171. doi: 10.1002/arch.940030206

Liu, S. S., Li, A. Y., Witt, C. M., and Perez de Leon, A. A. (2011). Immunohistological localization of serotonin in the CNS and feeding system of the stable fly Stomoxys calcitrans L. (Diptera: Muscidae). Arch. Insect Biochem. Physiol. 77, 199-219. doi: 10.1002/arch.20434

Ma, Y., Li, J. J., Tang, Q. B., Zhang, X. N., Zhao, X. C., Yan, F. M., et al. (2016). Trans-generational desensitization and within-generational resensitization of a sucrose-best neuron in the polyphagous herbivore Helicoverpa armigera (Lepidoptera: Noctuidae). Sci. Rep. 6:39358. doi: 10.1038/srep39358

Muscedere, M. L., Johnson, N., Gillis, B. C., Kamhi, J. F., and Traniello, J. F. A. (2012). Serotonin modulates worker responsiveness to trail pheromone in the ant Pheidole dentata. J. Comp. Physiol. A Neuroethol. Sens. Neural Behav. Physiol. 198, 219-227. doi: 10.1007/s00359-011-0701-2

Nässel, D. R. (1988). Serotonin and serotonin-immunoreactive neurons in the nervous system of insects. Prog. Neurobiol. 30, 1-85. doi: 10.1016/03010082(88)90002-0

Nässel, D. R., and Cantera, R. (1985). Mapping of serotonin-immunoreactive neurons in the larval nervous system of the flies Calliphora erythrocephala and Sarcophaga bullata. A comparison with ventral ganglion in adult animals. Cell Tissue Res. 239, 423-434. doi: 10.1007/bf00218023

Nässel, D. R., Ohlsson, L., and Sivasubramanian, P. (1987). Postembryonic differentiation of serotonin-immunoreactive neurons in fleshfly optic lobes developing in situ or cultured in vivo without eye discs. J. Comp. Neurol. 255, 327-340. doi: 10.1002/cne.902550302

Orchard, I. (2006). Serotonin: a coordinator of feeding-related physiological events in the blood-gorging bug, Rhodnius prolixus. Comp. Biochem. Physiol. A Mol. Integr. Physiol. 144, 316-324. doi: 10.1016/j.cbpa.2005.11.010

Page, T. L. (1987). Serotonin phase-shifts the circadian rhythm of locomotor activity in the cockroach. J. Biol. Rhythms. 2, 23-34. doi: 10.1177/ 074873048700200103

Paulk, A. C., Dacks, A. M., and Gronenberg, W. (2009). Color processing in the medulla of the bumblebee (Apidae: Bombus impatiens). J. Comp. Neurol. 513, 441-456. doi: 10.1002/cne.21993
Pfeiffer, K., and Homberg, U. (2014). Organization and functional roles of the central complex in the insect brain. Annu. Rev. Entomol. 59, 165-184. doi: 10.1146/annurev-ento-011613-162031

Pfuhl, G., Zhao, X. C., Ian, E., Surlykke, A., and Berg, B. G. (2014). Sound-sensitive neurons innervate the ventro-lateral protocerebrum of the heliothine moth brain. Cell Tissue Res. 355, 289-302. doi: 10.1007/s00441-0131749-9

Qi, Y.-X., Wu, S.-F., Huang, J., and Ye, G.-Y. (2014). Advances in 5-hydroxytryptamine and its receptors in insects. Acta Entomol. Sin. 57, 844-859. doi: 10.16380/j.kcxb.2014.07.005

Rehder, V., Bicker, G., and Hammer, M. (1987). Serotonin-immunoreactive neurons in the antennal lobe and suboesophageal ganglion of the honeybee. Cell Tissue Res. 247, 59-66. doi: 10.1007/bf00216547

Rogers, S. M., and Ott, S. R. (2015). Differential activation of serotonergic neurons during short- and long-term gregarization of desert locusts. Proc. Biol. Sci. 282:20042062. doi: 10.1098/rspb.2014.2062

Saifullah, A. S. M., and Tomioka, K. (2002). Serotonin sets the day state in the neurons that control coupling between the optic lobe circadian pacemakers in the cricket Gryllus bimaculatus. J. Exp. Biol. 205, 1305-1314.

Schoofs, A., Hückesfeld, S., and Pankratz, M. J. (2018). Serotonergic network in the subesophageal zone modulates the motor pattern for food intake in Drosophila. J. Insect Physiol. 106, 36-46. doi: 10.1016/j.jinsphys.2017. 07.007

Schürmann, F. W., and Klemm, N. (1984). Serotonin-immunoreactive neurons in the brain of the honeybee. J. Comp. Neurol. 225, 570-580. doi: 10.1002/cne. 902250407

Seidel, C., and Bicker, G. (1996). The developmental expression of serotoninimmunoreactivity in the brain of the pupal honeybee. Tissue Cell 28, 663-672. doi: 10.1016/s0040-8166(96)80070-X

Settembrini, B. P., and Villar, M. J. (2004). Distribution of serotonin in the central nervous system of the blood-feeding heteropteran, Triatoma infestans (Heteroptera: Reduviidae). J. Morphol. 260, 21-32. doi: 10.1002/jmor. 10211

Sitaraman, D., LaFerriere, H., Birman, S., and Zars, T. (2012). Serotonin is critical for rewarded olfactory short-term memory in Drosophila. J. Neurogenet. 26, 238-244. doi: 10.3109/01677063.2012.666298

Sitaraman, D., Zars, M., LaFerriere, H., Chen, Y. C., Sable-Smith, A., Kitamoto, T., et al. (2008). Serotonin is necessary for place memory in Drosophila. Proc. Nat. Acad. Sci. U S A 105, 5579-5584. doi: 10.1073/pnas.0710168105

Sun, X. J., Tolbert, L. P., and Hildebrand, J. G. (1993). Ramification pattern and ultrastructural characteristics of the serotonin-immunoreactive neuron in the antennal lobe of the moth Manduca sexta: a laser scanning confocal and electron microscopic study. J. Comp. Neurol. 338, 5-16. doi: 10.1002/cne. 903380103

Tang, Q.-B., Zhan, H., Berg, B.-G., Yan, F.-M., and Zhao, X.-C. (2014). Three dimensional reconstructions of the brain and the suboesophageal ganglion of Helicoverpa armigera (Lepidoptera: Noctuidae) Larvae. Acta Entomol. Sin. 57, 538-546. doi: 10.16380/j.kcxb.2014.05.003

Tomioka, K. (1999). Light and serotonin phase-shift the circadian clock in the cricket optic lobe in vitro. J. Comp. Physiol. 185, 437-444. doi: 10.1007/s003590 050404

Tyrer, N. M., Turner, J. D., and Altman, J. S. (1984). Identifiable neurons in the locust central nervous system that react with antibodies to serotonin. J. Comp. Neurol. 227, 313-330. doi: 10.1002/cne.902270303

Vallés, A. M., and White, K. (1988). Serotonin-containing neurons in Drosophila melanogaster: development and distribution. J. Comp. Neurol. 268, 414-428. doi: $10.1002 / \mathrm{cne} .902680310$

van der Woude, E., and Smid, H. M. (2017). Maximized complexity in miniaturized brains: morphology and distribution of octopaminergic, dopaminergic and serotonergic neurons in the parasitic wasp, Trichogramma evanescens. Cell Tissue Res. 369, 477-496. doi: 10.1007/s00441-0172642-8

Vleugels, R., Velinden, H., and Vanden Broeck, J. (2015). Serotonin, serotonin receptors and their actions in insects. Neurotransimitter 2:e314. doi: $10.14800 /$ nt.314

Wang, Y., Ma, Y., Zhou, D. S., Gao, S. X., Zhao, X. C., Tang, Q. B., et al. (2017). Higher plasticity in feeding preference of a generalist than a specialist: 
experiments with two closely related Helicoverpa species. Sci. Rep. 7:17876. doi: 10.1038/s41598-017-18244-7

Wegerhoff, R. (1999). GABA and Serotonin immunoreactivity during postembryonic brain development in the beetle Tenebrio molitor. Microsc. Res. Tech. 45, 154-164. doi: 10.1002/(sici)1097-0029(19990501)45:3<154::aidjemt $3>3.0 . \mathrm{co} ; 2-5$

Xie, G.-Y., Zhao, X.-C., Guo, P., Chen, Q.-Y., Wu, G.-L., Li, G.-P., et al. (2016). Anatomical structure of the central nervous system of Apolygus lucorum (Hemiptera: MIridae). Acta Entomol. Sin. 59, 446-455. doi: 10.16380/j.kcxb. 2016.04.010

Yuan, Q., Lin, F., Zheng, X., and Sehgal, A. (2005). Serotonin modulates circadian entrainment in Drosophila. Neuron 47, 115-127. doi: 10.1016/j.neuron.2005. 05.027

Zhao, X. C., and Berg, B. G. (2009). Morphological and physiological characteristics of the serotonin-immunoreactive neuron in the antennal lobe of the male oriental tobacco budworm, Helicoverpa assulta. Chem. Senses 34, 363-372. doi: 10.1093/chemse/bjp013

Zhao, X. C., Chen, Q. Y., Guo, P., Xie, G. Y., Tang, Q. B., Guo, X. R., et al. (2016). Glomerular identification in the antennal lobe of the male moth Helicoverpa armigera. J. Comp. Neurol. 524, 2993-3013. doi: 10.1002/cne. 24003
Zhao, X. C., Xie, G. Y., Berg, B. G., Schachtner, J., and Homberg, U. (2017). Distribution of tachykinin-related peptides in the brain of the tobacco budworm Heliothis virescens. J. Comp. Neurol. 525, 3918-3934. doi: $10.1002 / \mathrm{cne} .24310$

Zhou, D. S., van Loon, J. J. A., and Wang, C. Z. (2010). Experiencebased behavioral and chemosensory changes in the generalist insect herbivore Helicoverpa armigera exposed to two deterrent plant chemicals J. Comp. Physiol. A Neuroethol. Sens. Neural Behav. Physiol. 196, 791-799. doi: 10.1007/s00359-010-0558-9

Conflict of Interest Statement: The authors declare that the research was conducted in the absence of any commercial or financial relationships that could be construed as a potential conflict of interest.

Copyright (c) 2019 Tang, Song, Chang, Xie, Chen and Zhao. This is an open-access article distributed under the terms of the Creative Commons Attribution License (CC BY). The use, distribution or reproduction in other forums is permitted, provided the original author(s) and the copyright owner(s) are credited and that the original publication in this journal is cited, in accordance with accepted academic practice. No use, distribution or reproduction is permitted which does not comply with these terms. 